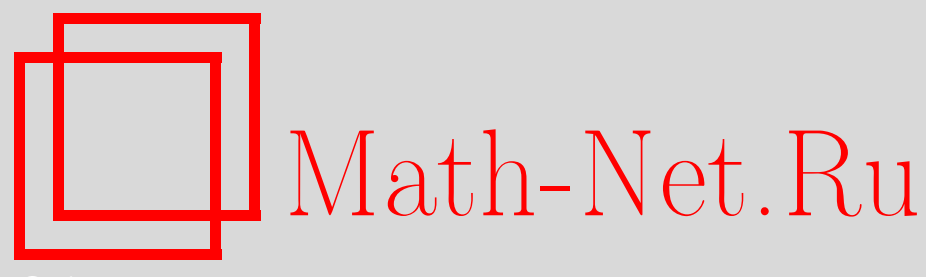

Х. Хоссейнхани, В. Файяз, С. А. А. Терохид, Н. Азими, 3. Зареи, М. Ганжи, Анизотропная космология при взаимодействии дилатонного поля и духовой темной энергии, ТМФ, 2018, том 194, номер 3, 481-509

DOI: https://doi.org/10.4213/tmf9323

Использование Общероссийского математического портала Math-Net.Ru подразумевает, что вы прочитали и согласны с пользовательским соглашением http://www.mathnet.ru/rus/agreement

Параметры загрузки:

IP : 54.174 .149 .18

26 апреля 2023 г., 17:29:54

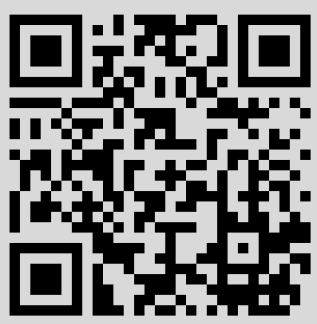




\title{
ФИЗИКА
}

Том 194, № 3

март, 2018

(C) 2018 г. $\quad$ Х. Хоссейнхани*, В. Файяз*, С. А. А. Терохид*, Н. Азими ${ }^{\dagger}$, 3. Зареи*, М. Ганжи*

\section{АНИЗОТРОПНАЯ КОСМОЛОГИЯ \\ ПРИ ВЗАИМОДЕЙСТВИИ ДИЛАТОННОГО ПОЛЯ И ДУХОВОЙ ТЕМНОЙ ЭНЕРГИИ}

\begin{abstract}
Изучается взаимодействие дилатонного скалярного поля с духовой темной энергией в анизотропной Вселенной. Эволюция темной энергии, которая доминирует во Вселенной, может быть полностью описана единственным дилатонным скалярным полем. Данная связь позволяет восстановить кинетическую энергию, а также динамику дилатонного скалярного поля, опираясь на эволюцию плотности энергии. На основании последних данных наблюдений получены ограничения для моделей духовой темной энергии, представляющих собой объединение моделей темной материи и темной энергии. Для этого исследовано, как при помощи наблюдаемых величин определить эволюцию расширения $H(z)$. Рассчитана эволюция возмущений плотности в линейном режиме как для духовой темной энергии, так и для обобщенной духовой темной энергии; результат сравнивается с моделями $\Lambda$ CDM. Обсуждается обоснованность обобщенного второго закона термодинамики во Вселенной Бьянки типа I. Полученная модель устойчива при поздних временах, но неустойчива для малых времен.
\end{abstract}

Ключевые слова: анизотропная Вселенная, духовая и обобщенная духовая темная энергия, дилатонное скалярное поле, обобщенный второй закон термодинамики, теория возмущений.

DOI: https://doi.org/10.4213/tmf9323

\section{1. ВВЕДЕНИЕ}

Космологические наблюдения за сверхновыми типа Іа показывают ускорение расширения Вселенной в нынешнюю эпоху [1], [2]. Кроме того, результаты, касающиеся крупномасштабного распределения галактик, и наиболее точные данные по

${ }^{*}$ Department of Physics, Hamedan Branch, Islamic Azad University, Hamedan, Iran. E-mail: hossienhossienkhani@yahoo.com, fayaz_vahid@yahoo.com, a.terohid@iauh.ac.ir, zarei.ph@gmail.com, mohsen.ganji@gmail.com

${ }^{\dagger}$ Department of Mathematics, Hamedan Branch, Islamic Azad University, Hamedan, Iran. E-mail: azimi1379@yahoo.com 
реликтовому излучению, полученные прибором WMAP, подтверждают такое ускорение [3], [4]. Наиболее неожиданный и парадоксальный результат этих наблюдений заключается в том, что 4\% общей плотности энергии Вселенной приходится на барионную материю, $27 \%$ - на небарионную материю, почти $69 \%$ - на совершенно неизвестную составляющую с отрицательным давлением. В научной литературе эта составляющая с отрицательным давлением называется темной энергией (ТЭ). Она создает силу отталкивания и увеличивает текущее ускоренное расширение Вселенной. Известными примерами предшествующих подходов являются такие модели ТЭ, как квинтэссенция [5], [6], К-эссенция [7], [8], тахион [9], [10], фантом [11]-[13], духовый конденсат [14], [15], квинтом [16]-[18], голографическая ТЭ [19], эволюционная ТЭ [20], [21] и др. Детальный обзор моделей ТЭ приведен в работах [22], [23]. Основное объяснение ускорения расширения основано на модификации исходной теории гравитации на больших масштабах, например, $f(R)$-гравитация [24]-[27] и сценарии мира на бранах [28]-[31].

$\mathrm{K}$ этой обширной коллекции моделей принадлежат модели духовой темной энергии (ДТЭ) [32]-[38]. В них космологические постоянные возникают благодаря так называемым духам Венециано, или духам квантовой хромодинамики [37]. Духи Венециано являются нефизическими в квантовой теории поля в пространстве-времени Минковского, но приводят к важным и нетривиальным физическим эффектам в расширяющейся Вселенной, а эти эффекты обуславливают плотность энергии вакуума $\rho_{\Lambda} \sim \Lambda_{\mathrm{QCD}}^{3} H \sim\left(10^{-3} \text { эВ }\right)^{4}$. При $H \sim 10^{-33}$ эВ и $\Lambda_{\mathrm{QCD}} \sim 100$ эВ мы получаем точное значение силы, которая на сегодняшний день ускоряет Вселенную. Общая энергия вакуума духа Венециано в квантовой хромодинамике имеет вид $H+O\left(H^{2}\right)$ [39], [40].

Неустойчивость модели ДТЭ по отношению к возмущениям изучалась в работе [41]. Тогда скалярное поле ТЭ может рассматриваться как эффективное описание этой духовой теории. Широко обсуждалось в научной литературе восстановление ДТЭ в терминах скалярных полей. Недавно Шейхи [42] построил квинтэссенцию, тахион, К-эссенцию и модели дилатонного скалярного поля ТЭ при взаимодействии ТЭ с горизонтом Хаббла как инфракрасное обрезание в плоской Вселенной. Также рассматривался обобщенный второй закон термодинамики для модели ТЭ с другим инфракрасным обрезанием в неплоской Вселенной [43]. Согласно этому закону энтропия жидкости внутри горизонта в дополнение к энтропии, связанной с горизонтом событий, является неубывающей функцией времени. Неравновесная картина термодинамики рассматривалась на горизонте событий модели Фридмана-Робертсона-Уокера (ФРУ) [44]. Карами и Абдолмалеки [45] изучали обоснованность обобщенного второго закона гравитационной термодинамики в рамках $f(T)$-гравитации, т. е. в модифицированном телепараллелизме.

В космическом микроволновом фоновом излучении были обнаружены некоторые широкоугольные аномалии, нарушающие статистическую изотропность Вселенной [46]. Плоскостные модели Бьянки (однородные, но не обязательно изотропные), видимо, дают наиболее многообещающее объяснение таких аномалий. Яффе и др. [47] показали, что удаление компоненты Бьянки из данных прибора WMAP может объяснить некоторые широкоугольные аномалии и оставить Вселенную изотропной. Таким образом, Вселенная могла бы достичь достаточно анизотропной геометрии в космологических моделях вне зависимости от инфляции. Кроме того, подобные модели могут быть классифицированы в зависимости от того, когда 
появляется анизотропия - на ранней стадии или позже. Модели ранних стадий Вселенной можно изменить так, чтобы по завершении инфляции мы имели слабо анизотропную геометрию [48].

Космологическая модель Бьянки типа I (BI), представляющая собой двухкомпонентную смесь идеальной жидкости, и модели ТЭ изучалась в [49], [50]. Также модели ВІ в присутствии анизотропной ТЭ рассматривались в контексте геометрии Лиры [51], [52]. В работе [53] изучалась взаимодействующая модель ДТЭ с квинтэссенцией, тахионом, K-эссенцией и соответствующими скалярными полями в анизотропной Вселенной. Недавно Сингх и др. [54] проанализировали поведение скалярного поля и космологической постоянной в $f(R, T)$-теории гравитации для Вселенной ВI; подробности см. в [54]-[58]. При помощи моделей ВI с различных точек зрения изучались возможные эффекты анизотропии в ранней Вселенной [48].

Для проверки жизнеспособности нашей теории мы налагаем космологические ограничения на взаимодействующую ДТЭ (ВДТЭ) и обобщенную ДТЭ (ОДТЭ) в анизотропной Вселенной. Таким образом устанавливается соответствие между сценариями ВДТЭ и дилатонным скалярным полем в анизотропной Вселенной. В работах [59], [60] путем введения взаимодействия между ДТЭ, ОДТЭ и темной материей (TM) было обнаружено, что параметр уравнения состояния взаимодействующей ТЭ может пересекать фантомную линию. Однако авторы этих работ ограничивались случаем, когда параметр Вселенной $\Omega_{\Lambda}$ и параметр анизотропии $\Omega_{\sigma}$ постоянны, и пренебрегали зависимостью от времени величин $\Omega_{\Lambda}$ и $\Omega_{\sigma}$ в анизотропной Вселенной. В настоящей работе изучается влияние анизотропии на производную по времени уравнения движения моделей ВДТЭ и ОДТЭ.

Статья имеет следующую структуру. В разделе 2 представлена модель ДТЭ с дилатонным скалярным полем в анизотропной Вселенной. Раздел 3 посвящен построению моделей дилатонного скалярного поля со взаимодействующей ДТЭ и изучению корректности обобщенного второго закона термодинамики. В разделах 4 и 5 уравнения модели ВІ переформулированы для случаев наличия и отсутствия взаимодействующей ОДТЭ в моделях дилатонного скалярного поля и приведены рассуждения, касающиеся выполнения обобщенного второго закона термодинамики в модели с ОДТЭ и дилатонным скалярным полем для анизотропной Вселенной. В разделе 6 для получения ограничений на космологические параметры моделей $\Lambda$ CDM, ДТЭ и ОДТЭ используются результаты измерения величины $H(z)$. В разделе 7 обсуждается линейная эволюция возмущений в моделях ДТЭ и ОДТЭ с анизотропной космологией. В разделе 8 изучается динамика эффективного фантомного дилатона для модели ВI. Раздел 9 содержит обсуждение полученных результатов.

\section{2. МОДЕЛЬ ДИЛАТОННОЙ ДТЭ В АНИЗОТРОПНОЙ ВСЕЛЕННОЙ}

Пространственно-однородное и анизотропное пространство-время ВІ описывается метрикой

$$
d s^{2}=-d t^{2}+A^{2} d x^{2}+B^{2} d y^{2}+C^{2} d z^{2} .
$$

Скорости расширения по трем декартовым направлениям $x, y$ и $z$ определяются как $H_{X}=\dot{A} / A, H_{Y}=\dot{B} / B$ и $H_{Z}=\dot{C} / C$, где точкой обозначена производная по времени. В таких моделях они различны, но в пределе $H_{X}=H_{Y}=H_{Z}$ плоское 
изотропное расширение ФРУ восстанавливается. Среди моделей Бьянки модель ВI обладает плоской геометрией и отсутствие полной завихренности; напротив, компоненты сдвига возникают естественным образом.

Теперь предположим, что среда - это идеальная жидкость, тогда тензор энергии импульса имеет вид

$$
T_{\mu \nu}=(\rho+p) u_{\mu} u_{\nu}+p g_{\mu \nu},
$$

где $u^{\nu}-4$-вектор, удовлетворяющий условию $u^{\nu} u_{\nu}=-1$, а $\rho$ - полная энергия идеальной жидкости, $p$ - соответствующее давление. Мы рассматриваем $a=(A B C)^{1 / 3}$ как усредненный скалярный фактор модели BI, тогда обобщенный средний параметр Хаббла и уравнения поля в модели ВI для метрики (1) с учетом (2) принимают вид

$$
\begin{aligned}
H & =\frac{1}{3}\left(H_{X}+H_{Y}+H_{Z}\right)=\frac{1}{3}\left(\frac{\dot{A}}{A}+\frac{\dot{B}}{B}+\frac{\dot{C}}{C}\right)=\frac{\dot{a}}{a}, \\
H^{2} & =\frac{1}{3}\left(8 \pi G \rho_{\mathrm{tot}}+\sigma^{2}\right) \\
\dot{H} & =-4 \pi G\left(\rho_{\mathrm{tot}}+p_{\mathrm{tot}}\right)-\sigma^{2} \\
2 \sigma^{2} & =\sigma_{\mu \nu} \sigma^{\mu \nu}=\left(\frac{\dot{A}}{A}\right)^{2}+\left(\frac{\dot{B}}{B}\right)^{2}+\left(\frac{\dot{C}}{C}\right)^{2}-\frac{1}{3} \theta^{2},
\end{aligned}
$$

где $\rho_{\mathrm{tot}}=\rho_{\mathrm{m}}+\rho_{\Lambda}, p_{\mathrm{tot}}=p_{\mathrm{m}}+p_{\Lambda}, G$ - гравитационная постоянная Ньютона, $\sigma^{2}=(1 / 2) \sigma_{i j} \sigma^{i j}$, где $\sigma_{i j}=u_{i, j}+\left(u_{i ; k} u^{k} u_{j}+u_{j ; k} u^{k} u_{i}\right) / 2+\theta\left(g_{i j}+u_{i} u_{j}\right) / 3$ - тензор сдвига, который описывает скорость деформации потока материи, а $\theta=u_{; j}^{j}-$ скалярное расширение. Мы рассматриваем случай, когда сдвиг доминирует над другими полями материи: $\sigma^{2} \gg 8 \pi G \rho_{\mathrm{tot}}$. С другой стороны, известно, что сдвиг меняется как $\sigma \propto a^{-3}$. Таким образом, следуя уравнению ВI, в эпоху доминирования сдвига Вселенная расширяется как $a \propto t^{1 / 3}$.

Хорошо известно, что динамические модели ТЭ играют ключевую роль в описании ускоренного расширения Вселенной. Модель ДТЭ Венециано - одна из динамических моделей ТЭ, определяемая как [33]

$$
\rho_{\Lambda}=\alpha H
$$

где $\alpha$ - постоянная, имеющая размерность [энергия $]^{3}$ и примерно порядок $\Lambda_{\mathrm{QCD}}^{3}$, где $\Lambda_{\mathrm{QCD}} \sim 100 \mathrm{MэB.} \mathrm{Используя} \mathrm{(4),} \mathrm{безразмерный} \mathrm{параметр} \mathrm{плотности} \mathrm{можно}$ определять стандартным образом:

$$
\Omega_{\mathrm{m}}=\frac{\rho_{\mathrm{m}}}{\rho_{\mathrm{cr}}}, \quad \Omega_{\Lambda}=\frac{\rho_{\Lambda}}{\rho_{\mathrm{cr}}}=\frac{\alpha}{3 M_{\mathrm{P}}^{2} H}, \quad \Omega_{\sigma}=\frac{\sigma^{2}}{3 H^{2}},
$$

где критическая плотность энергии $\rho_{\text {cr }}=3 M_{\mathrm{P}}^{2} H^{2}$, а масса Планка $M_{\mathrm{P}}^{2}=8 \pi G$. Используя эти уравнения и уравнение (4) модели ВI, получаем

$$
\Omega_{\mathrm{m}}+\Omega_{\Lambda}=1-\Omega_{\sigma}
$$

В настоящем разделе мы выводим общий вид уравнения состояния для плотности духовой энергии $\rho_{\Lambda}$ в пространстве-времени ВI без взаимодействия между плотностью ТЭ и холодной ТМ при $\omega_{\mathrm{m}}=0$. Таким образом, уравнения непрерывности 
для ТЭ и ТМ можно раздельно записать как

$$
\begin{aligned}
\dot{\rho}_{\Lambda}+3 H \rho_{\Lambda}\left(1+\omega_{\Lambda}\right) & =0, \\
\dot{\rho}_{\mathrm{m}}+3 H \rho_{\mathrm{m}} & =0
\end{aligned}
$$

где $\omega_{\Lambda}=p_{\Lambda} / \rho_{\Lambda}-$ параметр уравнения состояния для ТЭ. Теперь, взяв производную по времени от плотности ДТЭ (7) и используя уравнение ВI, получим

$$
\dot{\rho}_{\Lambda}=\rho_{\Lambda} \frac{\dot{H}}{H}=-\frac{\alpha}{2 M_{\mathrm{P}}^{2}}\left(2 M_{\mathrm{P}}^{2} \sigma^{2}+\rho_{\Lambda}\left(1+\omega_{\Lambda}+r\right)\right),
$$

где

$$
r=\frac{\rho_{\mathrm{m}}}{\rho_{\Lambda}}=\frac{1-\Omega_{\sigma}-\Omega_{\Lambda}}{\Omega_{\Lambda}}
$$

- отношение плотностей энергии. Подставляя соотношение (12) в уравнение непрерывности (10) и используя (9), находим параметр уравнения состояния ДТЭ:

$$
\omega_{\Lambda}=\frac{-1+\Omega_{\sigma}}{2-\Omega_{\Lambda}} .
$$

При ранних временах, когда $\Omega_{\Lambda} \ll 1$, имеем $\omega_{\Lambda}=\left(-1+\Omega_{\sigma}\right) / 2$. С другой стороны, при больших временах доминирует ТЭ, $\Omega_{\Lambda} \rightarrow 1$ и $\Omega_{\sigma} \rightarrow 0$, и ДТЭ ведет себя как космологическая постоянная с $\omega_{\Lambda}=-1$, которая входит в фазу де Ситтера в далеком будущем. Возьмем производную по времени от величины $\Omega_{\Lambda}(8)$ и используем соотношение $\dot{\Omega}_{\Lambda}=H \Omega_{\Lambda}^{\prime}$, получим уравнение движения ДТЭ в виде

$$
\Omega_{\Lambda}^{\prime}=\frac{3 \Omega_{\Lambda}}{2-\Omega_{\Lambda}}\left(1-\Omega_{\Lambda}+\Omega_{\sigma}\right),
$$

где штрих обозначает производную по $N=\ln a$.

Теперь мы можем установить соответствие между ДТЭ и дилатонным скалярным полем. В системе координат Эйнштейна действие принимает вид [61]

$$
S_{\mathrm{E}}=\int d^{4} x \sqrt{-g}\left[\frac{M_{\mathrm{P}}^{2}}{2} R-\gamma(\partial \phi)^{2}-V(\phi)\right]+S_{\mathrm{m}}\left(\phi, g_{\mu \nu, \psi}\right),
$$

где $\gamma$ - обобщенная постоянная дилатонного взаимодействия $(\gamma=2$ для типичных теорий суперструн), $V(\phi)$ - дилатонный потенциал. Инфляционные космологические модели периода до Большого взрыва, основанные на действии (16), рассматривались в работах [62], [63]. Гасперини и Риччи [62] получили точные решения для низкоэнергетического четырехмерного эффективного струнного действия, пользуясь дилатоном, не зависящим от положения в пространстве. Ниже мы анализируем некоторые общие свойства канонического космологического скалярного поля $\phi$, лагранжиан которого есть общая функция от скалярного квадратичного кинетического члена $\left(\partial \phi^{2}\right)$ и дилатонного скалярного поля. Действие дилатон-гравитации задается как [64]

$$
S_{\mathrm{D}}=\int d^{4} x \sqrt{-g}\left[\frac{M_{\mathrm{P}}^{2}}{2} R+p_{\mathrm{D}}(\chi, \phi)\right],
$$

где $\phi$ - так называемый дилатон, а параметр $\chi=-(1 / 2) \partial_{\mu} \phi \partial^{\mu} \phi$, который, как правило, также зависит от $\phi$. Действие дилатонного поля более общее, чем действие 
в теории Бранса-Дикке, где мы имеем дилатонный потенциал. Модель дилатонного скалярного поля также является интересной попыткой объяснить происхождение ТЭ с использованием теории струн. Дилатонное давление и плотность энергии задаются как [61], [65]

$$
\begin{aligned}
& p_{\mathrm{D}}=-\chi+c e^{\lambda \phi} \chi^{2}, \\
& \rho_{\mathrm{D}}=-\chi+3 c e^{\lambda \phi} \chi^{2},
\end{aligned}
$$

где $c$ и $\lambda$ - положительные постоянные. Эта модель возникает из четырехмерного эффективного струнного действия при низких энергиях и включает кинетические поправки более высоких порядков к древесному действию в низкоэнергетической эффективной струнной теории. Уравнения поля для действия (17) можно получить вариацией по полю $\phi$, что дает

$$
\ddot{\phi}\left(p_{\chi}+\dot{\phi}^{2} p_{\chi \chi}\right)+3 H p_{\chi} \dot{\phi}+2 \chi p_{\chi \phi}-p_{\phi}=-Q^{\prime}
$$

где $H$ определяется соотношением (3), $Q^{\prime}$ соответствует взаимодействию между материей и дилатоном, $p_{\chi}=d p / d \chi$ и $p_{\phi}=d p / d \phi$.

Рассмотрим дилатонный параметр уравнения состояния, который принимает вид

$$
\omega_{\mathrm{D}}=\frac{c e^{\lambda \phi} \chi-1}{3 c e^{\lambda \phi} \chi-1}
$$

В данном случае масштабирующее решение соответствует $\chi e^{\lambda \phi}=$ const, откуда $\phi \sim \ln t$. Условие ускоренного расширения дает $1 / 2<c e^{\lambda \phi} \chi<2 / 3$, и предел космологической постоянной соответствует $c e^{\lambda \phi} \chi=1 / 2$. Чтобы рассматривать дилатонное поле как эффективное описание духовой плотности, нужно использовать соответствие между дилатонной плотностью энергии и плотностью энергии духов. Это приводит к равенству $\rho_{\mathrm{D}}=\rho_{\Lambda}$. Приравнивая $(21)$ к (14), получаем решение

$$
c e^{\lambda \phi} \chi=\frac{3-\Omega_{\Lambda}-\Omega_{\sigma}}{5-\Omega_{\Lambda}-3 \Omega_{\sigma}}
$$

далее с учетом равенства $\chi=\dot{\phi}^{2} / 2$ имеем

$$
e^{\lambda \phi / 2} \dot{\phi}=\sqrt{\frac{2}{c}}\left(\frac{3-\Omega_{\Lambda}-\Omega_{\sigma}}{5-\Omega_{\Lambda}-3 \Omega_{\sigma}}\right)^{1 / 2} .
$$

Наконец, получаем

$$
\phi(a)-\phi(1)=\frac{2}{\lambda} \ln \left[\frac{3 M_{\mathrm{P}}^{2} \lambda}{\alpha \sqrt{c}} \int_{1}^{a} \Omega_{\Lambda}\left(\frac{3-\Omega_{\Lambda}-\Omega_{\sigma}}{5-\Omega_{\Lambda}-3 \Omega_{\sigma}}\right)^{1 / 2} \frac{d a}{a}\right]
$$

где мы взяли $a_{0}=1$ для настоящего времени, а $\Omega_{\Lambda}$ задано в (15). 


\section{3. МОДЕЛЬ ДИЛАТОННОЙ ВДТЭ В АНИЗОТРОПНОЙ ВСЕЛЕННОЙ}

В настоящем разделе рассматривается Вселенная, в которой взаимодействуют ТЭ и ТМ. Последние данные, полученные путем наблюдения галактических кластеров, также свидетельствуют в пользу взаимодействия между ТЭ и ТМ [66]. В присутствии взаимодействия уравнения непрерывности принимают вид

$$
\begin{aligned}
\dot{\rho}_{\Lambda}+3 H \rho_{\Lambda}\left(1+\omega_{\Lambda}\right) & =-Q, \\
\dot{\rho}_{\mathrm{m}}+3 H \rho_{\mathrm{m}} & =Q,
\end{aligned}
$$

где $Q$ - член взаимодействия, который может быть произвольной функцией от космологических параметров в терминах параметра Хаббла и плотностей энергии, $Q\left(H \rho_{\mathrm{m}}, H \rho_{\Lambda}\right)$. Итак, мы выбираем член взаимодействия следующего вида [67], [68]:

$$
Q=3 b^{2} H\left(\rho_{\mathrm{m}}+\rho_{\Lambda}\right)=3 b^{2} H \rho_{\Lambda}(1+r),
$$

где $b^{2}$ - постоянная взаимодействия. Положительный член $b^{2}$ калибрует переход энергии из ТЭ в ТМ. Отрицательный член $b^{2}$ нарушил бы второй закон термодинамики. Значение $b^{2}=0$ соответствует отсутствию взаимодействия, в то время как $b^{2}=1$ означает полный переход энергии из ТЭ в ТМ. Подставляя (12) и (27) в (25) и используя (13), получаем параметр уравнения состояния ВДТЭ:

$$
\omega_{\Lambda}=\frac{1}{2-\Omega_{\Lambda}}\left(-1+\Omega_{\sigma}+\frac{2 b^{2}}{\Omega_{\Lambda}}\left(-1+\Omega_{\sigma}\right)\right) .
$$

Можно легко проверить, что при поздних временах, когда $\Omega_{\Lambda} \rightarrow 1$ и $\Omega_{\sigma} \rightarrow 0$, параметр уравнения состояния ВДТЭ пересекает фантомную линию, а именно величина $\omega_{\Lambda}=-\left(1+2 b^{2}\right)<-1$ не зависит от значения постоянной взаимодействия $b^{2}$. В настоящем при выборе $\Omega_{\sigma 0}=0.001$ и $\Omega_{\Lambda}^{0}=0.69$ фантомное пересечение может быть получено, если $b^{2}>0.1$. Для прочих значений параметра анизотропии поведение параметра уравнения состояния при наличии и отсутствии взаимодействия ДТЭ показано на рис. 1а. Заметим, что параметр уравнения состояния для невзаимодействующих моделей ДТЭ всегда больше, чем $\omega_{\Lambda}=-1$, и остается в режиме квинтэссенции, т. е. $\omega_{\Lambda}>-1$. Однако при взаимодействии $\left(b^{2}>0\right)$ можно обнаружить, что $\omega_{\Lambda}$ может быть меньше, чем -1 . Это означает, что уравнение состояния $\omega_{\Lambda}$, возможно, пересекает фантомную линию во взаимодействующей модели ДТЭ. Также для заданного значения $\Omega_{\sigma 0}$ величина $\omega_{\Lambda}$ растет при малых временах и уменьшается при больших. Более того, для $\Omega_{\sigma 0}=0$ (модель $\left.Ф Р У\right) \omega_{\Lambda}$ стремится к -1 при больших значениях времени, в то время как для $\Omega_{\sigma 0} \neq 0$ параметр $\omega_{\Lambda}$ может пересечь фантомную линию. В присутствии взаимодействия эволюция ДТЭ задается следующим уравнением:

$$
\Omega_{\Lambda}^{\prime}=\frac{3 \Omega_{\Lambda}}{2-\Omega_{\Lambda}}\left(1-\Omega_{\Lambda}+\Omega_{\sigma}+b^{2}\left(-1+\Omega_{\sigma}\right)\right) .
$$

Динамика $\Omega_{\Lambda}$ при наличии взаимодействия ДТЭ и без него показана на рис. $1 б$. Мы видим, что для заданного значения $\Omega_{\sigma 0}$ величина $\Omega_{\Lambda}$ увеличивается при увеличении масштабирующего множителя, а при заданном масштабирующем множителе $a$ величина $\Omega_{\Lambda}$ уменьшается при увеличении $\Omega_{\sigma 0}$. Это согласуется с соотношением (9). Также мы видим, что увеличение (уменьшение) $b^{2}$ (соответственно $\Omega_{\sigma 0}$ ) 


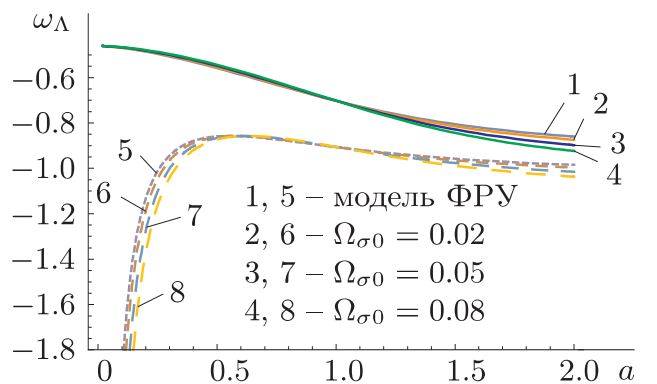

a

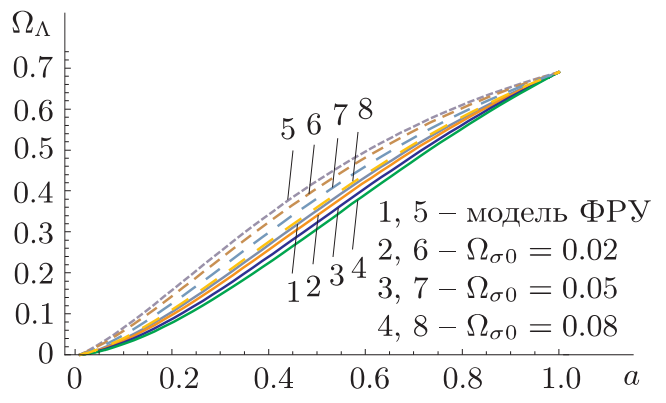

6

Рис. 1. Эволюция параметра уравнения состояния $\omega_{\Lambda}$ (а) и параметр плотности ТЭ $\Omega_{\Lambda}$ как функция коэффициента масштаба $а$ для разных параметров анизотропии $\Omega_{\sigma 0}$, рассматриваемых в настоящей работе (б). Невзаимодействующая ДТЭ обозначена сплошными линиями, ВДТЭ - штриховыми линиями, для $b^{2}=0$ и $b^{2}=0.1$ соответственно. В обоих случаях $\Omega_{\Lambda}^{0}=0.69$.

приводит к более крутым кривым зависимости параметров $\Omega_{\Lambda}$, отвечающих плотности ТЭ. Теперь мы используем связь между ВДТЭ и дилатонным скалярным полем. В этом случае кинетическую энергию можно получить как

$$
c e^{\lambda \phi} \chi=\frac{3-\Omega_{\Lambda}-\Omega_{\sigma}-\left(2 b^{2} / \Omega_{\Lambda}\right)\left(-1+\Omega_{\sigma}\right)}{5-\Omega_{\Lambda}-3 \Omega_{\sigma}-\left(6 b^{2} / \Omega_{\Lambda}\right)\left(-1+\Omega_{\sigma}\right)} .
$$

Наконец, интегрируя данное уравнение, получаем эволюционную форму поля:

$$
\phi(a)-\phi(1)=\frac{2}{\lambda} \ln \left[\frac{3 M_{\mathrm{P}}^{2} \lambda}{\alpha \sqrt{c}} \int_{1}^{a} \Omega_{\Lambda}\left(\frac{3-\Omega_{\Lambda}-\Omega_{\sigma}-\left(2 b^{2} / \Omega_{\Lambda}\right)\left(-1+\Omega_{\sigma}\right)}{5-\Omega_{\Lambda}-3 \Omega_{\sigma}-\left(6 b^{2} / \Omega_{\Lambda}\right)\left(-1+\Omega_{\sigma}\right)}\right)^{1 / 2} \frac{d a}{a}\right]
$$

где в настоящем мы берем $a_{0}=1$, а величина $\Omega_{\Lambda}$ задана в $(29)$. Ввиду сложности уравнений нельзя вывести аналитическое выражение для кинетической энергии, выраженной через фантомное дилатонное поле. Тем не менее можно получить это выражение численно.

Рис. 2а показывает, что скалярное поле $\phi$ возрастает до нуля в настоящий момент времени, что не похоже на другие модели ТЭ. Кроме того, из рисунка видно, что, во-первых, для заданной $\Omega_{\sigma 0}$ поле $\phi(a)$ уменьшается при увеличении масштабирующего множителя, а во-вторых, для заданного масштабирующего множителя поле $\phi(a)$ увеличивается при возрастании $\Omega_{\sigma 0}\left(b^{2}\right)$. На рис. 26 показано поведение дилатонной кинетической энергии как функции от $a$, видно, что эта энергия возрастает. Если дальнейшая эволюция кинетической энергии имеет поведение, соответствующее зеркальному отражению областей, приведенных на рис. 2, мы можем заключить, что уменьшение $\Omega_{\sigma 0}$ приводит к все более крутой кривой кинетической энергии. 

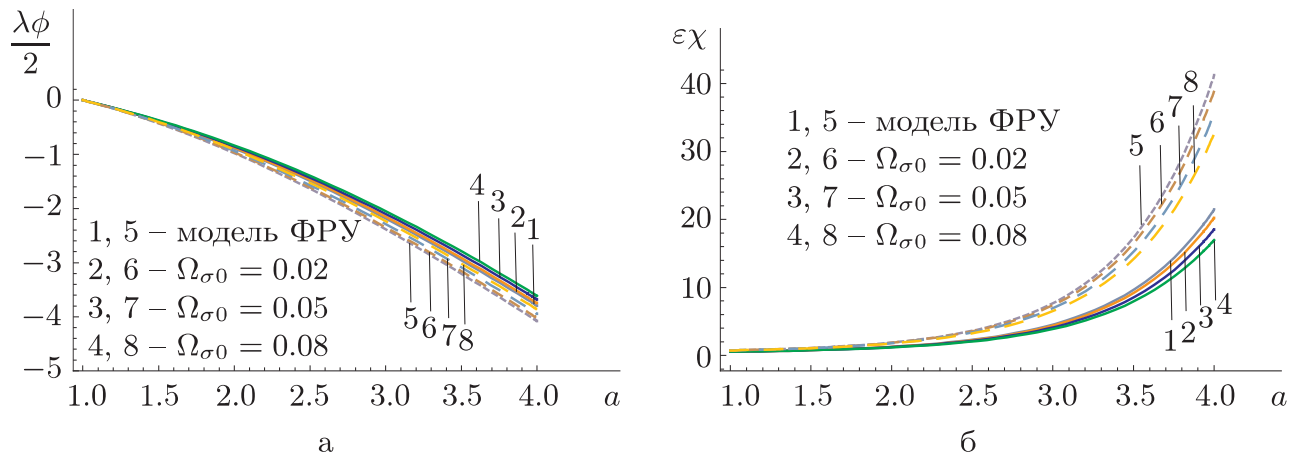

Рис. 2. Эволюция скалярного поля $\phi$ (а) и эволюция духовой дилатонной кинетической энергии $\chi=\dot{\phi}^{2} / 2$ (б) как функции масштабирующего множителя в случаях наличия и отсутствия взаимодействующей дилатонной ДТЭ для различных значений $\Omega_{\sigma 0}, \Omega_{\Lambda}^{0}=0.69, \phi(1)=0$ и $3 M_{\mathrm{P}}^{2} \lambda / \alpha \sqrt{c}=1$. Сплошные линии - отсутствие взаимодействия $\left(b^{2}=0\right)$; штриховые линии - наличие взаимодействия $\left(b^{2}=0.1\right)$.

Мы можем изучать устойчивость теории с помощью скорости звука ${ }^{1)}$ [69]

$$
c_{\mathrm{s}}^{2}=\frac{\dot{p}_{\mathrm{DG}}}{\dot{\rho}_{\mathrm{DG}}}=\frac{p_{\mathrm{DG}}^{\prime}}{\rho_{\mathrm{DG}}^{\prime}}
$$

где $p_{\mathrm{DG}}$ и $\rho_{\mathrm{DG}}$ определяются с помощью (18) и (19) соответственно. Используя соотношения (18), (19), (21), (25) и (30)-(32), получаем

$$
c_{\mathrm{s}}^{2}=-\frac{2\left(-1+\Omega_{\sigma}\right)\left[\Omega_{\Lambda}\left(-1+\Omega_{\Lambda}\right)+b^{2}\left(-4+3 \Omega_{\Lambda}\right)\right]}{\Omega_{\Lambda}\left(-2+\Omega_{\Lambda}\right)^{2}},
$$

что является квадратом скорости звука для ВДТЭ. Графический анализ величины $c_{\mathrm{s}}^{2}$ показывает, что наша теория может быть неустойчивой в ранние моменты времени, но на поздних стадиях она может быть устойчивой $\left(c_{\mathrm{s}}^{2} \geqslant 0\right)$ в зависимости от параметров модели.

На рис. 3 представлено поведение скорости звука в зависимости от масштабирующегно множителя при разных $\Omega_{\sigma 0}$. При фиксированных значениях прочих параметров увеличение значения $\Omega_{\sigma 0}$ повышает устойчивость теории на поздних стадиях эволюции. Более того, мы видим что сценарий без взаимодействия более устойчив, чем сценарий в присутствии взаимодействия.

\section{1. Дилатонная ВДТЭ с ТМ и обобщенный второй закон термодина-} мики в модели ВІ. Теперь проверим, выполняется ли обобщенный второй закон термодинамики для дилатонной ВДТЭ в анизотропной Вселенной. Бекенштейн [70] показал, что между энтропией горизонта черной дыры и окрестностью горизонта

\footnotetext{
1)В теории возмущений предполагается, что имеется малая флукутация плотности энергии, а далее определяется, будет ли это возмущение расти или исчезнет. В линейном приближении возмущенная плотность энергии имеет вид $\rho(t, \vec{x})=\rho(t)+\delta \rho(t, \vec{x})$, где $\rho(t)$ - невозмущенная плотность энергии.
} 


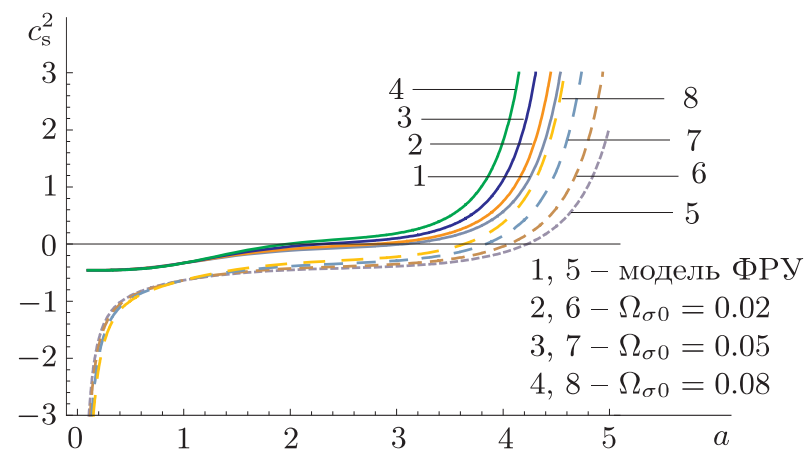

Рис. 3. Поведение квадрата скорости звука $c_{\mathrm{s}}^{2}$ как функции а для невзаимодействующей ДТЭ (сплошные линии) и ВДТЭ (штриховые линии) для модели ВI, $\Omega_{\Lambda}^{0}=0.69$.

имеется связь, которая играет важную роль при обсуждении обобщенного второго закона термодинамики. Закон гласит, что сумма энтропии черной дыры и энтропии фона должна увеличиваться с течением времени. В работе [71] исследовались термодинамические законы для космологических горизонтов, которые присутствуют во многих космологических моделях. Проверим, что сумма энтропий окрестности горизонта и самого горизонта, которую мы обозначим через $S_{\text {tot }}$ есть неубывающая функция времени. Данный принцип известен как обобщенный второй закон термодинамики; он записывается с помощью неравенства $\dot{S}_{\text {tot }} \geqslant 0$. Наша цель - ответить на вопрос, верно ли, что $\dot{S}_{\text {tot }} \geqslant 0$ при взаимодействующих дилатонной ДТЭ и ТМ в рамках модели ВI?

Первый закон термодинамики гласит, что

$$
T d S=d E+p d V
$$

где $T, S, p, V$ и $E$ обозначают температуру, энтропию, давление, объем и внутреннюю энергию системы соответственно. Дифференцируя по времени, получаем

$$
\dot{S}_{\mathrm{DG}}=\frac{\dot{E}_{\mathrm{DG}}+p_{\mathrm{DG}} \dot{V}}{T}, \quad \dot{S}_{\mathrm{m}}=\frac{\dot{E}_{\mathrm{m}}+p_{\mathrm{m}} \dot{V}}{T},
$$

где $S_{\mathrm{DG}}$ и $S_{\mathrm{m}}$ - энтропия дилатонных фантомных темных компонент и энтропия компонент материи соответственно. Внутренние энергии дилатонной ДТЭ и ТМ имеют вид

$$
E_{\mathrm{DG}}=\frac{4 \pi}{3} r_{A}^{3} \rho_{\mathrm{DG}}, \quad E_{\mathrm{m}}=\frac{4 \pi}{3} r_{A}^{3} \rho_{\mathrm{m}},
$$

где $r_{A}=H^{-1}$ есть радиус горизонта событий во Вселенной $\mathrm{BI}^{2)}$. Используя (35) и дифференцируя оба уравнения (36), получаем вариацию энтропии жидкости внутри горизонта событий:

$$
\dot{S}_{\mathrm{DG}}+\dot{S}_{\mathrm{m}}=\frac{4 \pi r_{A}^{2}}{T_{\mathrm{DG}}}\left[\dot{r}_{A} \rho_{\Lambda}\left(1+\omega_{\mathrm{DG}}\right)+\frac{r_{A}}{3} \dot{\rho}_{\mathrm{DG}}\right]+\frac{4 \pi r_{A}^{2}}{T_{\mathrm{m}}}\left[\dot{r}_{A} \rho_{\mathrm{m}}+\frac{r_{A}}{3} \dot{\rho}_{\mathrm{m}}\right] .
$$

2) Напомним, что в случае модели ФРУ $(k \neq 0)$, как и в нашем случае, горизонт событий задается как $r_{A}=1 / \sqrt{H^{2}+k^{2} / a^{2}}$. 


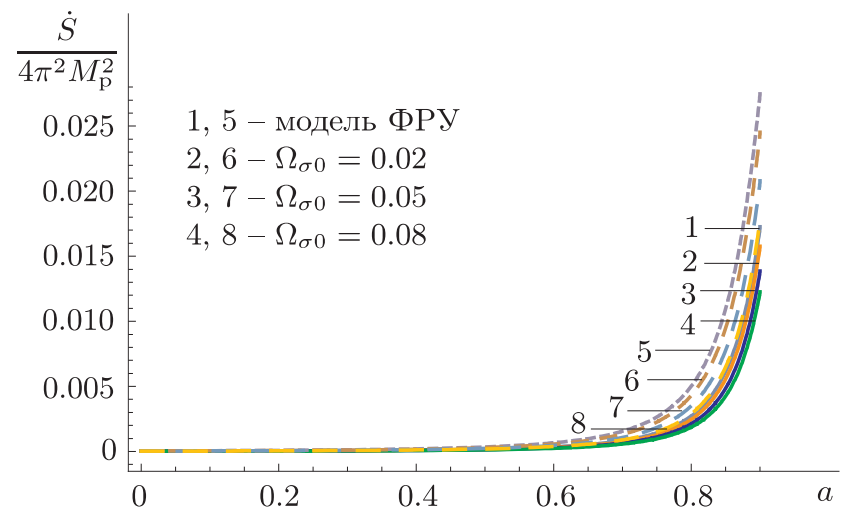

Рис. 4. Эволюция второго закона термодинамики $\dot{S}_{\text {tot }}$ как функция $a$ при различных $\Omega_{\sigma 0}$. Штриховые линии - ВДТЭ при $b^{2}=0.1, \Omega_{\Lambda}^{0}=0.69$ и $H_{0}=$ $72 \mathrm{KM}^{-1} \mathrm{Mпк}^{-1}$. Сплошные линии - ВДТЭ $\left(b^{2}=0\right)$.

В состоянии термодинамического равновесия предполагается, что все присутствующие во Вселенной жидкости имеют одну температуру $T$, в то время как их энергии и давления могут в общем случае различаться. Если рассматривать равновесие общей плотности энергии, как предложено в работе [72] для моделей ТЭ, температуры темного сектора при поздних временах удовлетворяют условию $T_{\mathrm{DG}} \approx T_{\mathrm{m}}$. Это предположение, несмотря на то что оно не является общепринятым [73], [74], будет использоваться в нашей работе. Используя законы сохранения $(25),(26)$ и $\dot{H}$ из (12), перепишем уравнение (37) как

$$
\dot{S}_{\mathrm{DG}}+\dot{S}_{\mathrm{m}}=\frac{8 \pi M_{\mathrm{P}}^{2} r_{A}^{2}}{T}\left(-\dot{r}_{A}+r_{A} H\right)\left(\dot{H}+\sigma^{2}\right) .
$$

Теперь мы должны рассмотреть температуру жидкостей $T$, которая, кроме того, принимается равной температуре горизонта $T_{\mathrm{h}}$. Обобщим это на любую поверхность, связанную с ТЭ, как в случае дилатонной ДТЭ. Ее температура задана как $T_{\mathrm{h}}=1 / 2 \pi r_{A}$ (см. [72] и приведенную там литературу). Энтропия, связанная с горизонтом, имеет вид $S_{\mathrm{h}}=8 \pi^{2} M_{\mathrm{P}}^{2} r_{A}^{2}$. Для получения вариации полной энтропии добавим скорость изменения энтропии (38) с производной энтропии $S_{\mathrm{h}}$ по космическому времени:

$$
\frac{\dot{S}_{\mathrm{tot}}}{16 \pi^{2} M_{\mathrm{P}}^{2} r_{A}}=\dot{r}_{A}+r_{A}^{2}\left(-\dot{r}_{A}+H r_{A}\right)\left(\dot{H}+\sigma^{2}\right),
$$

где $\dot{S}_{\mathrm{tot}}=\dot{S}_{\mathrm{h}}+\dot{S}_{\mathrm{DG}}+\dot{S}_{\mathrm{m}}$. Знак выражения зависит только от $\left(H, r_{A}, \dot{\mathrm{r}}_{A}, \dot{H}\right)$ и верен вне зависимости от выбора горизонта для модели Вселенной, которую мы рассматриваем. Чтобы выполнялся обобщенный второй закон термодинамики, нужно, чтобы выпонялось условие $\dot{S}_{\mathrm{h}}+\dot{S}_{\mathrm{DG}}+\dot{S}_{\mathrm{m}} \geqslant 0$, т. е. сумма энтропии материи внутри горизонта не должна быть убывающей функцией времени. Используя тот факт, что в анизотропной Вселенной BI $\dot{r}_{A}=-\dot{H} / H^{2}$, и выражение для $\dot{H}(12)$, входящее в (39), получаем

$$
\frac{\dot{S}_{\mathrm{tot}}}{4 \pi^{2} M_{\mathrm{P}}^{2}}=\frac{3}{H}\left(4 \Omega_{\sigma}+3\left(1+\Omega_{\sigma}+\Omega_{\mathrm{DG}} \omega_{\mathrm{DG}}\right)\left(1-\Omega_{\sigma}+\Omega_{\mathrm{DG}} \omega_{\mathrm{DG}}\right)\right) .
$$


Мы рассматриваем соотношения (18) и (19), а кинетическую энергию получаем из формулы (30) с членом взаимодействия (25). Производная по времени полной энтропии $\dot{S}_{\text {tot }}$ при наличии и отсутствии взаимодействия дилатонной ДТЭ изображен на рис. 4 при различных $\Omega_{\sigma 0}$. Получаем, что $\dot{S}_{\mathrm{tot}}>0$, и это демонстрирует справедливость обобщенного второго закона термодинамики. Также мы видим, что производная $\dot{S}_{\text {tot }}$ убывает с ростом $\Omega_{\sigma 0}$ для заданного $a$.

\section{4. МОДЕЛЬ НЕВЗАИМОДЕЙСТВУЮЩЕЙ ДИЛАТОННОЙ ДТЭ В АНИЗОТРОПНОЙ ВСЕЛЕННОЙ}

В настоящем разделе мы представляем основной сценарий для невзаимодействующей ОДТЭ с холодной ТМ в анизотропной Вселенной. В работе [75] показано, что использование члена $H^{2}$ для обычной ДТЭ может быть полезно для описания ранней эволюции Вселенной, которая определяется следующим образом:

$$
\rho_{\Lambda}=\alpha H+\beta H^{2}
$$

где $\beta$ - еще одна постоянная размерности [энергия] ${ }^{2}$. Кроме того, для безразмерного параметра плотности ОДТЭ получаем

$$
\Omega_{\Lambda}=\frac{\rho_{\Lambda}}{\rho_{\mathrm{cr}}}=\frac{\alpha+\beta H}{3 M_{\mathrm{P}}^{2} H} .
$$

Дифференцируя уравнение (41), имеем

$$
\dot{\rho}_{\Lambda}=\dot{H}(\alpha+2 \beta H) .
$$

Используя соотношения (10), (41) и (43), получаем параметр уравнения состояния:

$$
\omega_{\Lambda}=\frac{\xi-\Omega_{\Lambda}+\Omega_{\sigma}\left(\Omega_{\Lambda}+\xi\right)}{\Omega_{\Lambda}\left(2-\Omega_{\Lambda}-\xi\right)},
$$

где $\xi=\beta /\left(3 M_{\mathrm{P}}^{2}\right)$ и эволюция $\Omega_{\Lambda}$ и $H$ определяются ОДТЭ во Вселенной $\mathrm{BI}^{3)}$. Ясно, что данное соотношение сводится к соответствующему соотношению для Вселенной ФРУ с ДТЭ, когда $\xi=\sigma=0$ [76]. Принимаем $\xi=0$, тогда выражение для $\omega_{\Lambda}$ сводится к соотношению (14) при отсутствии взаимодействия. В дальнейшем мы реализуем соответствие между ОДТЭ и моделью дилатонного скалярного поля. Используя соотношения (18), (19), (21) и (44), можно легко показать, что эволюция обобщенного духового дилатонного скалярного поля и кинетической энергии принимает следующий вид:

$$
\begin{aligned}
c e^{\lambda \phi} \chi & =\frac{\xi\left(1+\Omega_{\sigma}\right)-\Omega_{\Lambda}\left(3-\Omega_{\Lambda}-\Omega_{\sigma}-\xi\right)}{3 \xi\left(1+\Omega_{\sigma}\right)-\Omega_{\Lambda}\left(5-\Omega_{\Lambda}-3 \Omega_{\sigma}-\xi\right)} \\
\phi(a)-\phi(1) & =\frac{2}{\lambda} \ln \left[\frac{\lambda}{\sqrt{c}} \int_{1}^{a} \frac{1}{H a}\left(\frac{\xi\left(1+\Omega_{\sigma}\right)-\Omega_{\Lambda}\left(3-\Omega_{\Lambda}-\Omega_{\sigma}-\xi\right)}{3 \xi\left(1+\Omega_{\sigma}\right)-\Omega_{\Lambda}\left(5-\Omega_{\Lambda}-3 \Omega_{\sigma}-\xi\right)}\right)^{1 / 2} d a\right] .
\end{aligned}
$$

3) Для плоской Вселенной ВІ, используя (4), (10), (41), (43) и (44), получаем

$$
\Omega_{\Lambda}^{\prime}=\frac{-3\left(\xi-\Omega_{\Lambda}\right)\left(1-\Omega_{\Lambda}+\Omega_{\sigma}\right)}{2-\Omega_{\Lambda}-\xi}, \quad H^{\prime}=-3 H\left(\frac{1+\Omega_{\sigma}-\Omega_{\Lambda}}{2-\Omega_{\Lambda}-\xi}\right) .
$$




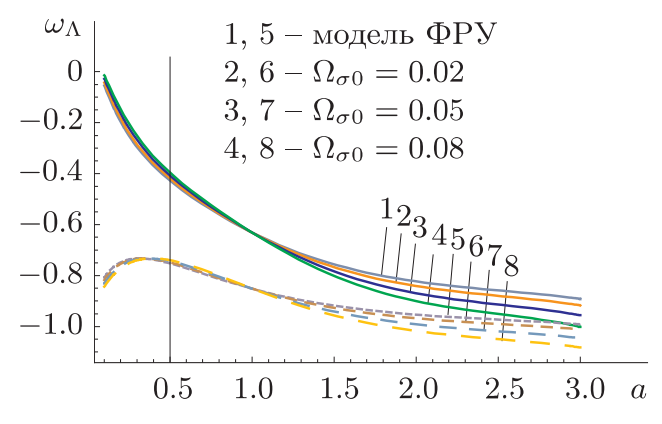

a

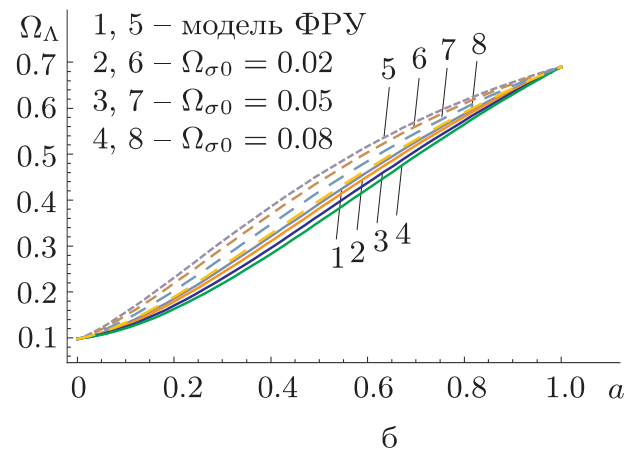

Рис. 5. Поведение $\omega_{\Lambda}$ (а) и $\Omega_{\Lambda}$ (б) для взаимодействующей ОДТЭ и невзаимодействующей ОДТЭ и различных значений $\Omega_{\sigma 0}$. Сплошные линии отвечают отсутствию взаимодействия $\left(b^{2}=0\right)$, штриховые - наличию $\left(b^{2}=0.1\right)$. В обоих случаях $\Omega_{\Lambda}^{0}=0.69, \xi=0.1$.

\section{5. МОДЕЛЬ ВЗАИМОДЕЙСТВУЮЩЕЙ ДИЛАТОННОЙ ДТЭ В АНИЗОТРОПНОЙ ВСЕЛЕННОЙ}

Чтобы включить в модель взаимодействие с холодной ТМ в анизотропной Вселенной, мы расширяем модель ОДТЭ. Используя формулу (27) для $Q$ и подставляя (43) в (25), получаем параметр уравнения состояния взаимодействующей ОДТЭ:

$$
\omega_{\Lambda}=\frac{\xi-\Omega_{\Lambda}+\Omega_{\sigma}\left(\Omega_{\Lambda}+\xi\right)-2 b^{2}\left(1-\Omega_{\sigma}\right)}{\Omega_{\Lambda}\left(2-\Omega_{\Lambda}-\xi\right)} .
$$

Проанализируем поведение параметра уравнения состояния при трех различных значениях $\Omega_{\sigma 0}$. На рис. 5 показано поведение $\omega_{\Lambda}$ и $\Omega_{\Lambda}$ в зависимости от масштабирующего множителя $a$ при разных $\Omega_{\sigma 0}$. На всех рисунках в настоящей работе мы обозначаем взаимодействующую ОДТЭ как ВДТЭ, а невзаимодействующую ОДТЭ как ОДТЭ. Рис. 5а показывает, что в случае ОДТЭ значения параметра уравнения состояния, отвечающие настоящему моменту, начинаются в период, когда доминирует материя, и достигают основной области квинтэссенции. Но в случае взаимодействующей ОДТЭ мы видим, что параметр уравнения состояния начинается в области высокой квинтэссенции и спускается к низшим отрицательным значениям фазы квинтэссенции, а потом поворачивает в сторону области духов, пересекая эпоху, когда доминирует вакуум, для всех параметров анизотропии. Из рис. $5 б$ следует, что $\Omega_{\Lambda}$ уменьшается при увеличении $\Omega_{\sigma 0}$. В частности, эволюция $\Omega_{\Lambda}$ для ОДТЭ без взаимодействия $\left(b^{2}=0\right)$ также показана на рис. 5 , и величина $\Omega_{\Lambda}$ при наличии взаимодействия больше, чем без взаимодействия.

Теперь восстановим динамику скалярного поля и соответствующие члены кинетической энергии в зависимости от постоянной взаимодействия $b^{2}$ во Вселенной ВI. Приравнивая $\omega_{\Lambda}$ к параметру уравнения состояния взаимодействующей ОДТЭ (47), находим

$$
c e^{\lambda \phi} \chi=\frac{\xi\left(1+\Omega_{\sigma}\right)-\Omega_{\Lambda}\left(3-\Omega_{\Lambda}-\Omega_{\sigma}-\xi\right)-2 b^{2}\left(1-\Omega_{\sigma}\right)}{3 \xi\left(1+\Omega_{\sigma}\right)-\Omega_{\Lambda}\left(5-\Omega_{\Lambda}-3 \Omega_{\sigma}-\xi\right)-6 b^{2}\left(1-\Omega_{\sigma}\right)} .
$$




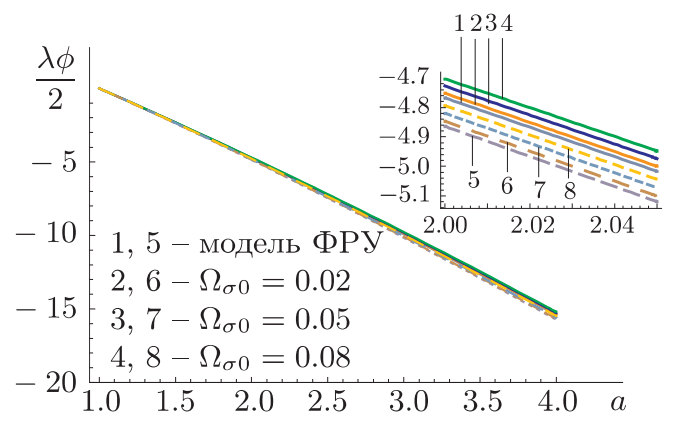

a

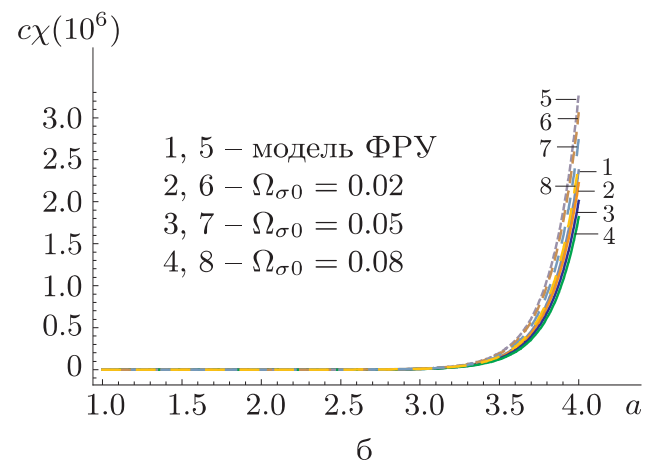

Рис. 6. Графики $\phi($ а) и $\chi(б)$ в зависимости от показателя масштаба в дилатонной взаимодействующей ОДТЭ (ОДТЭ). Сплошные линии соответствуют ОДТЭ $\left(b^{2}=0\right)$, а штриховые - взаимодействующей ОДТЭ $\left(b^{2}=0.1\right)$. В обоих случаях $\Omega_{\Lambda}^{0}=0.69, \phi(1)=0, H_{0}=72, \xi=0.1$ и $\lambda / \sqrt{c}=1$.

Используя уравнение (48) и уравнение $\dot{\phi}^{2}=2 \chi$, получаем обобщенное фантомное скалярное поле

$$
\begin{aligned}
& \phi(a)-\phi(1)=\frac{2}{\lambda} \times \\
& \times \ln \left[\frac{\lambda}{\sqrt{c}} \int_{1}^{a} \frac{1}{H a}\left(\frac{\xi\left(1+\Omega_{\sigma}\right)-\Omega_{\Lambda}\left(3-\Omega_{\Lambda}-\Omega_{\sigma}-\xi\right)-2 b^{2}\left(1-\Omega_{\sigma}\right)}{3 \xi\left(1+\Omega_{\sigma}\right)-\Omega_{\Lambda}\left(5-\Omega_{\Lambda}-3 \Omega_{\sigma}-\xi\right)-6 b^{2}\left(1-\Omega_{\sigma}\right)}\right)^{1 / 2} d a\right]
\end{aligned}
$$

где $\Omega_{\Lambda}$ и $H$ задаются взаимодействующей ОДТЭ во Вселенной $\mathrm{BI}^{4)}$.

Дилатонное поле $\phi$ и потенциал $\chi$ как функции параметра $a$ показаны на рис. 6 , начальное условие выбрано в виде $\phi(1)=0$, а прочие параметры оставлены такими же, как в предыдущем разделе. Из рис. 6а видно, что скалярное поле уменьшается со временем, а потенциал $\chi$ демонстрирует противоположное поведение. Более того, легко убедиться, что соответствующие кривые эволюции для различных значений параметра анизотропии пересекаются. Это означает, что значения параметра анизотропии $\Omega_{\sigma 0}$ не оказывают почти никакого влияния на эволюционные траектории поля $\phi$ при фиксированном значении $\xi$. Рис. $6 б$ показывает, что $\chi$ может возрастать при увеличении $a$, т. е. чем выше $\Omega_{\sigma 0}$, тем медленнее изменяется $\chi$. Более того, $\chi$ для ОДТЭ со взаимодействием растет быстрее, чем без взаимодействия.

Теперь изучим устойчивость взаимодействующей модели ОДТЭ во Вселенной ВI. С помощью соотношений (32), (41) и (48), (49) после несложных алгебраических вычислений получаем

$c_{\mathrm{s}}^{2}=-2 \times$

$$
\times \frac{\Omega_{\Lambda}\left(\Omega_{\Lambda}-1\right)\left(\Omega_{\sigma}-1\right)+\xi\left[\Omega_{\Lambda}-1+\Omega_{\sigma}\left(-3+2 \Omega_{\Lambda}\right)\right]+\xi^{2} \Omega_{\sigma}+b^{2}\left(\Omega_{\sigma}-1\right)\left(-4+3 \Omega_{\Lambda}+\xi\right)}{\left(\Omega_{\Lambda}+\xi\right)\left(-2+\Omega_{\Lambda}+\xi\right)^{2}},
$$

\footnotetext{
4) Берем производную обеих частей уравнения ВI (4) относительно космического времени и, используя уравнения $(9),(25),(27),(37),(42)$ и $(47)$, получаем $\Omega_{\Lambda}$ и $H$ :

$$
\Omega_{\Lambda}^{\prime}=\frac{-3\left(\xi-\Omega_{\Lambda}\right)\left(1-\Omega_{\Lambda}+\Omega_{\sigma}-b\left(1-\Omega_{\sigma}\right)\right)}{2-\Omega_{\Lambda}-\xi}, \quad H^{\prime}=-3 H\left(\frac{1+\Omega_{\sigma}-\Omega_{\Lambda}-b\left(1-\Omega_{\sigma}\right)}{2-\Omega_{\Lambda}-\xi}\right) .
$$
}




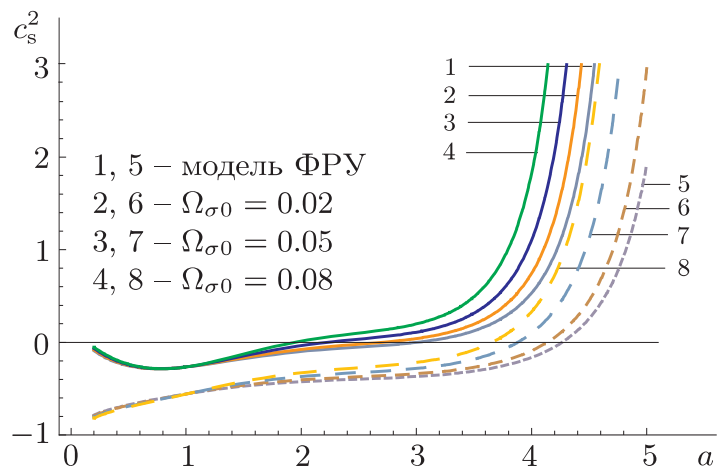

Рис. 7. Эволюция квадрата скорости звука $c_{\mathrm{s}}^{2}$ при изменении $а$ для модели взаимодействующей ОДТЭ. Обозначения те же, что на рис. 5.

что дает скорость звука для взаимодействующей дилатонной ОДТЭ во Вселенной ВІ. Эволюция величины $c_{\mathrm{s}}^{2}$ по параметру а показана на рис. 7. Анализ поведения величины $c_{\mathrm{s}}^{2}$ показывает, что наша теория может быть неустойчивой на ранних временах, но на поздних стадиях она может быть устойчива $\left(c_{\mathrm{s}}^{2}>0\right)$ в зависимости от значений параметров модели. Важным моментом является чувствительность устойчивости к параметрам $\Omega_{\sigma 0}$ и $b^{2}$. Возрастание $\Omega_{\sigma 0}$ при $b^{2}=0$ ведет к большей устойчивости к возмущениям.

Наконец, исследуем, справедлив ли обобщенный второй закон термодинамики для взаимодействующей ОДТЭ во Вселенной ВІ. Используя соотношения (25), (27), $(35),(36),(41),(42)$ и (47), получаем производную по времени полной энтропии для взаимодействующей дилатонной ОДТЭ:

$$
\begin{aligned}
& \dot{S}_{\text {tot }}=\frac{48 \pi^{2} M_{\mathrm{P}}^{2}}{H} \times \\
& \times\left[\Omega_{\sigma}+\frac{3\left(1-\Omega_{\Lambda}+\Omega_{\sigma}-b^{2}\left(1-\Omega_{\sigma}\right)\right)\left(\xi \Omega_{\sigma}+\left(-1+\Omega_{\sigma}\right)\left(-1+b^{2}+\Omega_{\Lambda}\right)\right)}{\left(-2+\Omega_{\Lambda}+\xi\right)^{2}}\right] .
\end{aligned}
$$

Производная $\dot{S}_{\text {tot }}$ полной энтропии по времени как функция масштабирующего множителя $a$ для взаимодействующей и невзаимодействующей ОДТЭ представлена на рис. 8 для различных значений $\Omega_{\sigma 0}$. Видно, что $\dot{S}_{\text {tot }}>0$ для всех возможных значений $\Omega_{\sigma 0}$, и это доказывает справедливость обобщенного второго закона термодинамики для дилатонного скалярного поля ОДТЭ.

\section{6. КОСМОЛОГИЧЕСКАЯ ЭВОЛЮЦИЯ ПАРАМЕТРА ХАББЛА ДЛЯ ДУХОВОЙ И ОБОБЩЕННОЙ МОДЕЛЕЙ ДТЭ}

В настоящем разделе мы используем результаты измерения параметра Хаббла в зависимости от величины красного смещения для получения ограничений на космологические параметры. Параметр Хаббла зависит от дифференциального возраста Вселенной как функция красного смещения [77]:

$$
H(z)=-\frac{1}{1+z} \frac{d z}{d t}
$$




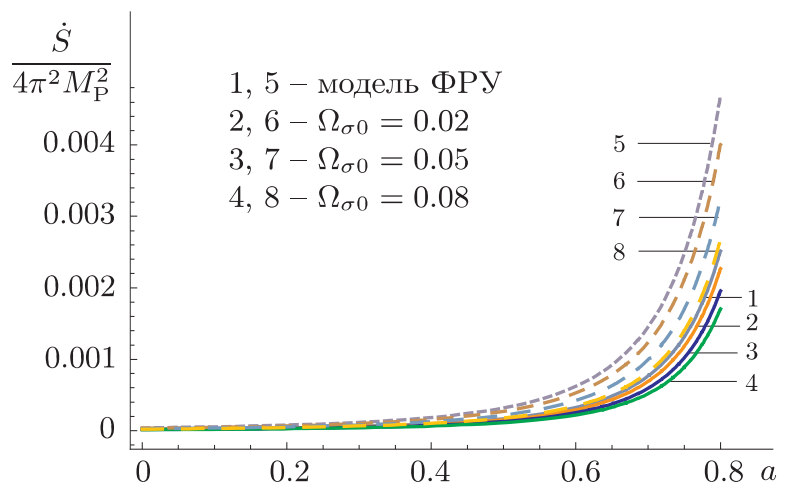

Рис. 8. Эволюция обобщенного второго закона термодинамики $\dot{S}_{\text {tot }}$ при изменении $а$ для модели дилатонной ОДТЭ при наличии и отсутствии взаимодействия для различных $\Omega_{\sigma 0}$. Здесь $\Omega_{\Lambda}^{0}=0.69, \xi=0.1, H_{0}=72$. Обозначения те же, что на рис. 5.

Так как поведение красного смещения $z$ во времени известно с высокой точностью, дифференциальное измерение времени $(d t)$ при заданном красном смещении позволяет непосредственно и точно измерить $H(z)$. В последние годы было приложено много усилий для вычисления $H(z)$. На текущий момент в нашем распоряжении имеется более 30 исследований $H(z)$ с использованием двух различных измерительных методов. Первый метод был предложен в работе [77]. В настоящей работе мы используем текущие и будущие данные для $H(z)$, чтобы одним и тем же способом получить ограничения для трех моделей ТЭ - ДТЭ, ОДТЭ и $\Lambda$ CDM. Заметим, что мы рассматриваем здесь плоскую Вселенную ВI, поэтому уравнение ВI (4) может быть представлено как

$$
H=H_{0}\left(\frac{\Omega_{m 0}(1+z)^{3}+\Omega_{\sigma 0}(1+z)^{6}}{1-\Omega_{\Lambda}}\right)^{1 / 2},
$$

где $H_{0}, \Omega_{m 0}$ и $\Omega_{\sigma 0}$ - текущие значения $H, \Omega_{\mathrm{m}}$ и $\Omega_{\sigma}$. Величина $H(z)$ обычно выражается в единицах км с ${ }^{-1} \mathrm{Mnк}^{-1}$. В модели $\Lambda$ CDM параметр Хаббла

$$
H=H_{0}\left(\Omega_{m 0}(1+z)^{3}+\Omega_{\sigma 0}(1+z)^{6}+\Omega_{\Lambda}\right)^{1 / 2}
$$

и уравнение состояния ТЭ есть $\omega_{\Lambda}=-1$. Текущая величина Согласно космологическим наблюдениям текущее значение составляет $\Omega_{\Lambda}=0.73 \pm 0.04$ [78]. В космологии $\Lambda \mathrm{CDM}$ выбрано значение $\Omega_{m 0}=0.274$, которое получается по данным пятилетних наблюдений WMAP [79]. Также для такой модели, как $w \mathrm{CDM}$ (модель с постоянным параметром уравнения состояния $w$ ),

$$
H=H_{0}\left(\Omega_{m 0}(1+z)^{3}+\Omega_{\sigma 0}(1+z)^{6}+\left(1-\Omega_{m 0}-\Omega_{\sigma 0}\right)(1+z)^{3(1+w)}\right)^{1 / 2} .
$$

По данным измерений реликтового излучения и барионных акустических осцилляция [80] текущие предпочтительные значения составляют $w=-1.01 \pm 0.15$ [78], $w=-0.98 \pm 0.12[3]$ и $w=-1.13_{-0.25}^{+0.24}$. Как и в случае феноменологической модели, включающей взаимодействие, в нашем сценарии взаимодействие между ОДТЭ 


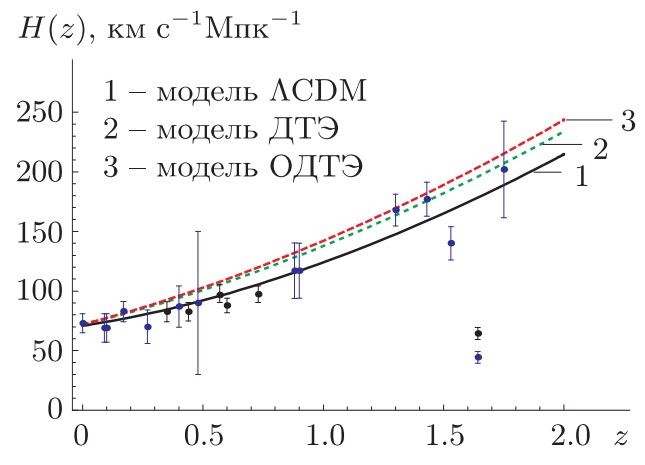

a

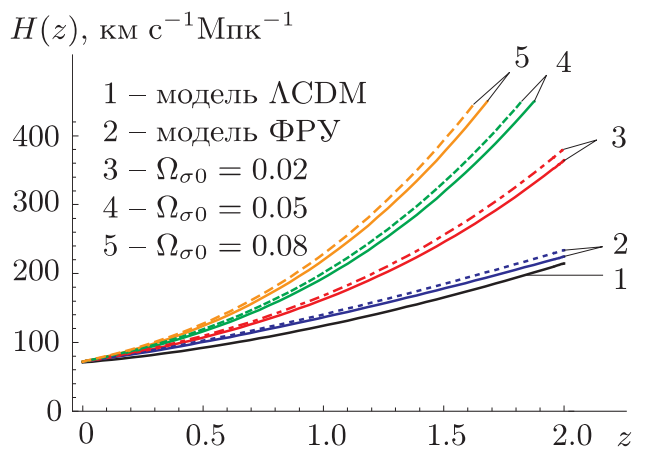

б

Рис. 9. Сравнение наблюдаемого параметра $H(z)$ как функции красного смещения $z$ для трех моделей - $\Lambda$ CDM, ДТЭ и ОДТЭ - при $b^{2}=0.1$, $H_{0}=72 \mathrm{Kм} \mathrm{c}^{-1} \mathrm{Mпк}^{-1}$ [4], $\Omega_{m 0}=0.277$ [84] и $\xi=0.1$. Модель ДТЭ показана сплошными линиями, а ОДТЭ - штриховыми. Данные представлены с погрешностями, изображенными отрезками в точках графика, линии являются теоретическими для различных моделей ТЭ и наблюдаемого $H(z)$.

(ДТЭ) и ТМ может быть описано при помощи параметра $b^{2}$ как феноменологическое взаимодействие. Экспериментальные результаты налагают ограничения на среднее значение параметра взаимодействия: по наблюдениям реликтового излучения и барионных акустических осцилляций $b^{2}=-0.61_{-0.25}^{+0.12}$ [81], по данным реликтового излучения и космического телескопа Хаббла [82] $b^{2}=-0.67_{-0.17}^{+0.086}$, а согласно данным по искривлению пространства из-за красного смещения [83] получается, что $b^{2}=0.00328_{-0.00328-0.00328-0.00328}^{+0.000736+0.00549+0.00816}$. Теперь, с учетом ДТЭ, используя соотношения $(8),(9)$ и $(53)$, имеем

$$
H=H_{0}\left(\frac{1-\Omega_{m 0}-\Omega_{\sigma 0}}{2}+\sqrt{\frac{1}{4}\left(1-\Omega_{m 0}-\Omega_{\sigma 0}\right)^{2}+\Omega_{m 0}(1+z)^{3}+\Omega_{\sigma 0}(1+z)^{6}}\right) .
$$

Уравнение Хаббла моделей ОДТЭ задано как

$$
\begin{aligned}
H= & H_{0}\left(\frac{1-\xi-\Omega_{m 0}-\Omega_{\sigma 0}}{2(1-\xi)}+\right. \\
& \left.+\sqrt{\frac{1}{4}\left(\frac{1-\xi-\Omega_{m 0}-\Omega_{\sigma 0}}{1-\xi}\right)^{2}+\frac{1}{1-\xi}\left(\Omega_{m 0}(1+z)^{3}+\Omega_{\sigma 0}(1+z)^{6}\right)}\right),
\end{aligned}
$$

где постоянная Хаббла $H_{0}$ выбрана равной $72 \mathrm{kм} \mathrm{c}^{-1} \mathrm{Mnк}^{-1}$. Имеются другие измерения постоянной Хаббла: $H_{0}=73.8 \pm 2.4 \mathrm{kм} \mathrm{c}^{-1} \mathrm{Mпк}^{-1}[84], H_{0}=73 \pm 3 \mathrm{kм} \mathrm{c}^{-1} \mathrm{M} п к^{-1}$ согласно данным прибора WMAP [4] и $H_{0}=68 \pm 4 \mathrm{kм} \mathrm{c}^{-1} \mathrm{M}^{-1}$ согласно усредненному статистическому анализу, выполненному на основе 461 измерений $H_{0}$ [85], [86].

На рис. 9 показана эволюция постоянной Хаббла как функции космического красного смещения z для различных космологических моделей ДТЭ, ОДТЭ и $\Lambda$ CDM. Для наложения ограничений на космологические параметры мы используем данные по $H(z)$ [85]-[90]. Параметр Хаббла в данных моделях больше, чем во Вселенной $\Lambda$ CDM. Это означает, что величина ускорения наибольшая для моделей ОДТЭ 


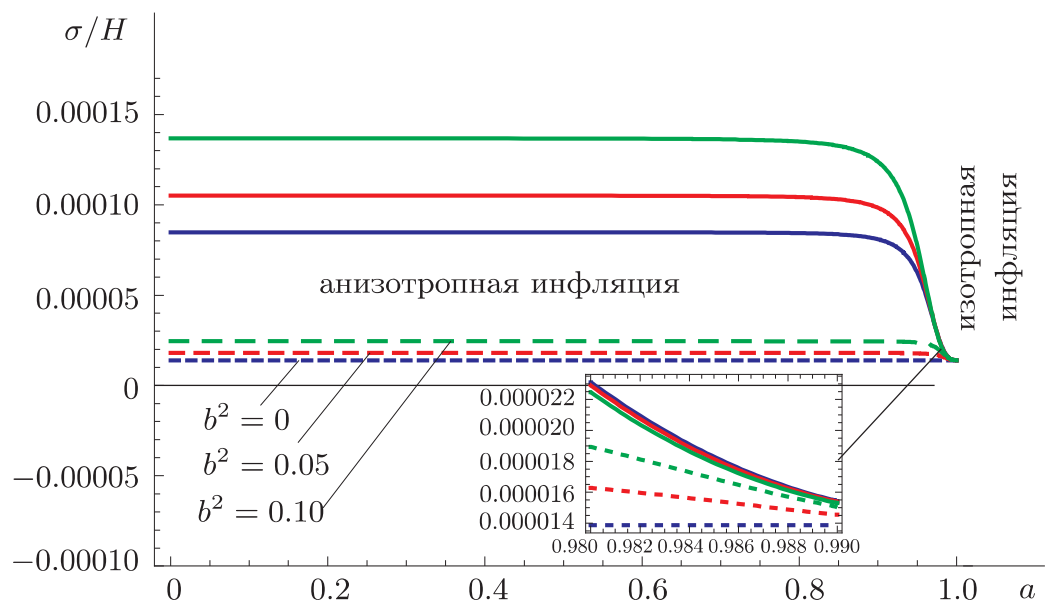

Рис. 10. Эволюция параметра анизотропии $\sigma / H$ по масштабному фактору $а$ для взаимодействующей ДТЭ (штриховые линии) и ОДТЭ (сплошные линии) при различных значениях $b^{2}$ и $\sigma_{0}=0.05$ [91]. Параметр становится практически постоянным в фазе анизотропной инфляции. Далее он стремительно убывает до величины $\mathcal{O}(\sigma \mathcal{H})$.

(ДТЭ) и что модель, в которой жидкость играет роль ТЭ, важна на протяжении длительного периода космической истории. Также согласно рис. 9, чем больше выбранное значение $\Omega_{\sigma 0}$, тем больше значение расширения Хаббла $H(z)$.

Наконец, рассмотрим изотропизацию модели. Мера анизотропии описывается параметром $\sigma / H$, т. е. величиной сдвига в пространстве-времени относительно средней скорости расширения. Тем не менее на рис. 10 мы видим, что в течение инфляционной фазы влияние сдвига не может быть больше параметра Хаббла, и сдвиг уменьшается к концу инфляции.

\section{7. ТЕОРИЯ ЛИНЕЙНЫХ ВОЗМУЩЕНИЙ ДЛЯ АНИЗОТРОПНОЙ ВСЕЛЕННОЙ}

Мы изучаем линейный рост возмущений для различных значений масштаба в эру доминирования материи, рассчитывая эволюцию фактора роста $g(a)$ в космологиях ДТЭ и ОДТЭ и сравнивая результат с решением, полученным для модели $\Lambda$ CDM. Дифференциальное уравнение для $g(a)$ имеет вид [92]-[94]

$$
g^{\prime \prime}(a)+\left(\frac{3}{a}+\frac{E^{\prime}(a)}{E(a)}\right) g^{\prime}(a)-\frac{2}{3} \frac{\Omega_{\mathrm{m} 0}}{a^{5} E^{2}(a)} g(a)=0,
$$

где $E=H / H_{0}$. В модели ТЭ без взаимодействия, используя соотношения (53)-(55), численно решаем уравнение (56) с целью изучения линейного роста в моделях ВI с ДТЭ и ОДТЭ . Далее мы сравниваем линейный рост для моделей ДТЭ и ОДТЭ с линейным ростом в модели $\Lambda$ CDM. Для получения начальных условий, так как мы рассматриваем линейный режим, фактор линейного роста имеет степенное решение $g(a) \propto a^{n}$ при $n>1$, тогда линейный рост увеличивается с течением времени. 

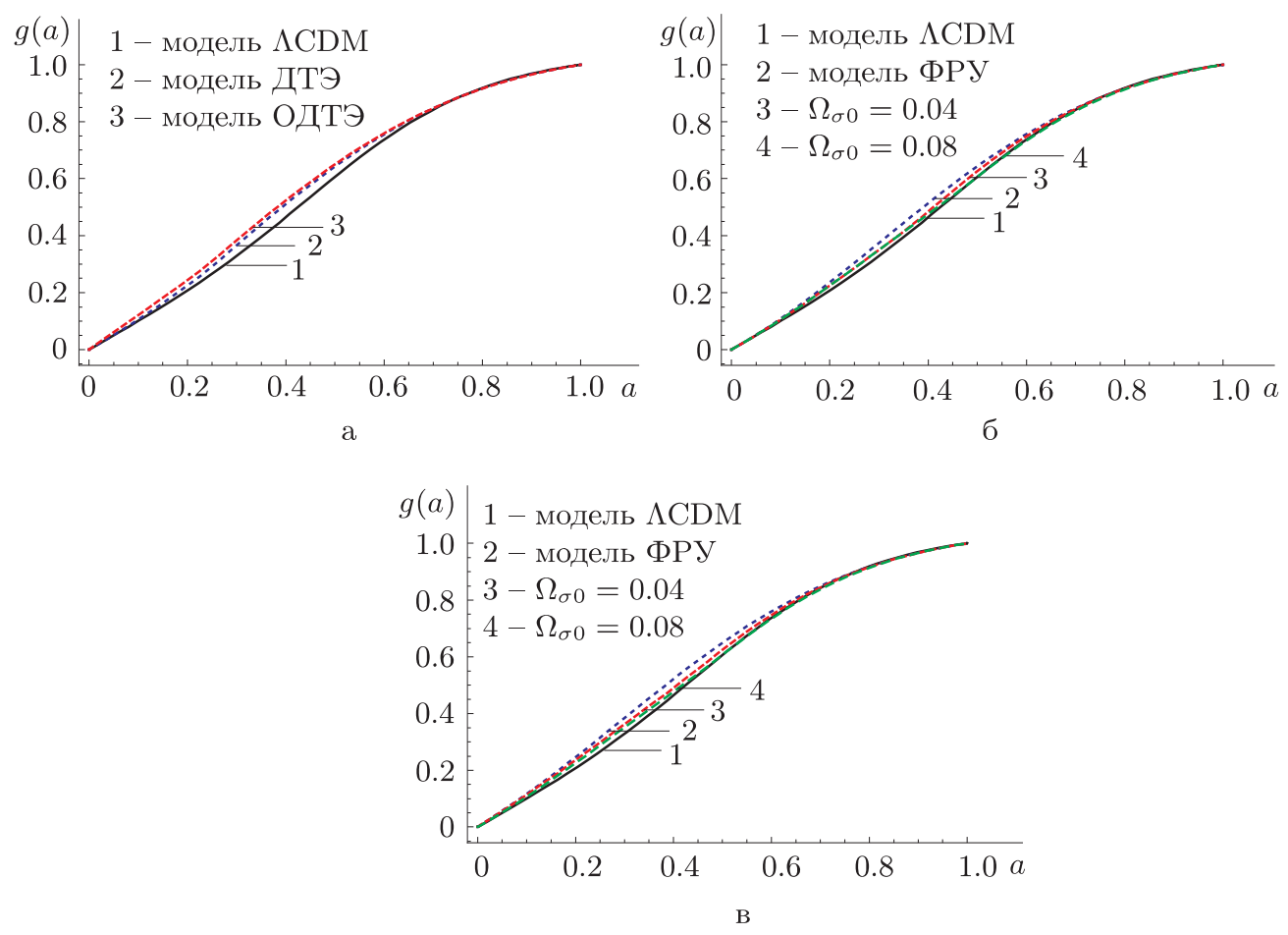

Рис. 11. Эволюция с течением времени фактора роста как функции показателя масштаба для различных космологических моделей в анизотропной Вселенной. Для удобства сравнения моделей мы фиксируем вариацию для модели ОДТЭ (ДТЭ) $\left(\Omega_{\sigma 0}=0.001\right)$, как показано на рис. $11 \mathrm{a}, \xi=0.1$.

На рис. 11 представлена эволюция фактора роста $g(a)$.Все модели нормированы так, чтобы они совпадали при ранних временах. Из рис. 11а видно, что фактор роста в модели ОДТЭ больше, чем в ДТЭ и $\Lambda$ CDM. Это можно объяснить, учитывая эволюцию параметра Хаббла на рис. 9. Параметр Хаббла больше в анизотропной Вселенной моделей ОДТЭ (ДТЭ), а наименьшее расширение наблюдается в модели ФРУ. Таким образом, фактор роста $g(a)$ для ОДТЭ (ДТЭ) всегда меньше во Вселенной ФРУ. Но это верно для моделей $\Lambda$ CDM. На рис. 11б, в мы видим, что для модели ФРУ фактор роста меняется пропорционально масштабирующему множителю, как мы и ожидали. В случаях ДТЭ (ОДТЭ) с $\Omega_{\sigma 0} \neq 0$ (анизотропная Вселенная) $g(a)$ меняется медленнее, чем в модели ФРУ $\left(\Omega_{\sigma 0}=0\right)$. В модели $\Lambda$ CDM заметно, что эволюция $g(a)$ протекает медленнее, чем в случае анизотропной Вселенной.

\section{8. АНАЛИЗ УСТОЙЧИВОСТИ МОДЕЛИ ДИЛАТОННОГО СКАЛЯРНОГО ПОЛЯ ДЛЯ МОДЕЛИ ВІ}

Для рассмотрения задачи квантовой устойчивости скалярного поля с действием (17), разделим скалярное поле $\phi$ на однородную часть $\phi_{0}$ и колебание $\delta \phi[14]:$

$$
\phi(t, \vec{x})=\phi_{0}(t)+\delta \phi(t, \vec{x}) .
$$


Разлагая $p(\chi, \phi)$ до второго порядка по $\delta \phi$, легко найти лагранжиан и гамильтониан колебаний. Гамильтониан имеет вид [95]

$$
\mathcal{H}=\zeta_{1} \frac{(\delta \dot{\phi})^{2}}{2}+\zeta_{2} \frac{(\nabla \delta \phi)^{2}}{2}-p_{\phi \phi} \frac{(\delta \phi)^{2}}{2},
$$

где $\zeta_{1}=p_{\chi}+2 \chi p_{\chi \chi}$ и $\zeta_{2}=p_{\chi}$, а для устойчивости классических возмущений требуется соблюдение условий $\zeta_{1} \geqslant 0$ и $\zeta_{2} \geqslant 0$. Если записать лагранжиан $p(\chi, \phi)$ в виде $p(\chi, \phi)=-\chi-V(\phi)$, как в большей части моделей ДТЭ [12], то коэффициенты $\zeta_{1}$ и $\zeta_{2}$ будут отрицательными. Устойчивость непрерывного решения, которое стремится к неподвижной точке, приводит к устойчивости этой точки. Для моделей одного и двух полей достаточные условия устойчивости изотропных решений, стремящихся к неподвижным точкам, были получены в работах [96], [97]. Было обнаружено, что решения с фантомными скалярными полями могут быть устойчивы для модели ВI. В работе [98] данный факт был описан как приближение в рамках фундаментальной теории. Так как фундаментальная теория должна быть устойчива и должна допускать квантование, подобная неустойчивость должна рассматриваться как аномалия приближения.

Получим решения для исследуемой модели и определим их устойчивость. С этой целью изучим космологическую эволюцию фантомного дилатонного лагранжиана (18). Полевые уравнения, вытекающие из действия (17), можно записать как

$$
\begin{aligned}
& 3 H^{2}-\sigma^{2}=\rho_{\mathrm{m}}-\frac{1}{2} \dot{\phi}^{2}+\frac{3}{4} c e^{\lambda \phi} \dot{\phi}^{4}, \\
& 2 \dot{H}+2 \sigma^{2}=-\rho_{\mathrm{m}}+\dot{\phi}^{2}-c e^{\lambda \phi} \dot{\phi}^{4} .
\end{aligned}
$$

Чтобы превратить данные уравнения в систему автономных дифференциальных уравнений первого порядка, определим следующий набор нормированных переменных разложения [99]:

$$
\Sigma_{1}^{2}=\frac{\dot{\phi}^{2}}{6 H^{2}}, \quad \Sigma_{2}^{2}=\frac{e^{-\lambda \phi}}{3 H^{2}} .
$$

Это налагает условие на динамические переменные

$$
\Omega_{\phi}+\Omega_{\mathrm{m}}=1-\Omega_{\sigma}, \quad \Omega_{\phi}=-\Sigma_{1}^{2}+3 c \frac{\Sigma_{1}^{4}}{\Sigma_{2}^{2}} .
$$

Тогда из (59) и (60) получаем

$$
H^{\prime}=-\frac{3}{2} H\left(1-\Sigma_{1}^{2}+\Omega_{\sigma}+\frac{c \Sigma_{1}^{4}}{\Sigma_{2}^{2}}\right),
$$

где штрихом обозначена производная $d / d N$. Используя $(20)$ и $(63)$, получаем

$$
\begin{aligned}
\Sigma_{1}^{\prime} & =\frac{3 \Sigma_{1}}{2}\left[1-\Sigma_{1}^{2}+\Omega_{\sigma}+\frac{c \Sigma_{1}^{4}}{\Sigma_{2}^{2}}\right]- \\
& -\frac{3 \Sigma_{2}^{2}}{6 c \Sigma_{1}^{2}-\Sigma_{2}^{2}}\left[\Sigma_{1}\left(\frac{-\Sigma_{2}^{2}+2 c \Sigma_{1}^{2}}{\Sigma_{2}^{2}}\right)+\frac{\sqrt{6} c \lambda \Sigma_{1}^{4}}{2 \Sigma_{2}^{2}}+\frac{\sqrt{6} Q}{6}\left(1+\Sigma_{1}^{2}-\Omega_{\sigma}-\frac{c \Sigma_{1}^{4}}{\Sigma_{2}^{2}}\right)\right], \\
\Sigma_{2}^{\prime} & =-\frac{\sqrt{6}}{2} \lambda \Sigma_{1} \Sigma_{2}+\frac{3 \Sigma_{2}}{2}\left(1-\Sigma_{1}^{2}+\Omega_{\sigma}+\frac{c \Sigma_{1}^{4}}{\Sigma_{2}^{2}}\right)
\end{aligned}
$$




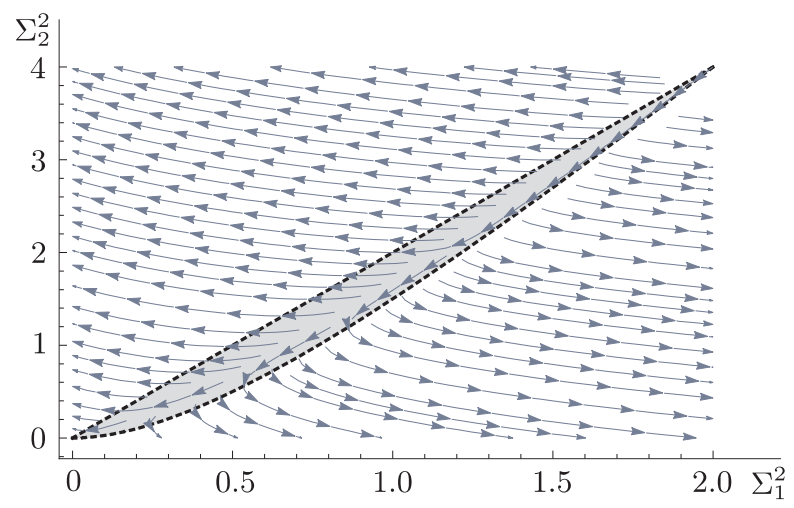

Рис. 12. Эволюция фазового пространства при $c=1$. Оба параметра должны находиться внутри области для того, чтобы модель была устойчива. Область устойчивости затемнена.

где $Q=Q^{\prime} / \rho_{\mathrm{m}}$. Далее мы получаем условия на новые переменные для поддержания устойчивости модели: $\Omega_{\phi}=-\Sigma_{1}^{2}+3 c \Sigma_{1}^{4} / \Sigma_{2}^{2} \leqslant 1-\Omega_{\sigma}$ и $p_{\chi} \geqslant 0$, что дает

$$
\Sigma_{2}^{2} \geqslant \frac{3 c \Sigma_{1}^{4}}{1+\Sigma_{1}^{2}-\Omega_{\sigma}}, \quad \Sigma_{2}^{2} \leqslant 2 c \Sigma_{1}^{2}
$$

На рис. 12 изображена область, описанная условиями (66), в координатах $\left(\Sigma_{2}^{2}, \Sigma_{1}^{2}\right)$. Из рисунка видно, что для поддержания устойчивости модели $\left(\Sigma_{2}^{2}, \Sigma_{1}^{2}\right)$ должны лежать внутри области.

Теперь, необходимо найти неподвижные точки системы, условие для которых имеет вид [100]

$$
\Sigma^{\prime}=\mathbf{f}(\Sigma)
$$

где $f$ определяется из (64), (65). Мы лишь рассмотрим динамическую систему переменных $\boldsymbol{\Sigma} \equiv\left(\Sigma_{1}, \Sigma_{2}\right)$, определенных уравнениями $(64),(65)$. Неподвижная точка $\boldsymbol{\Sigma}^{*}$ задается условием

$$
\mathbf{f}\left(\Sigma^{*}\right)=0 .
$$

Характер динамической системы определен собственными значениями матрицы

$$
\left.\frac{\partial \mathbf{f}}{\partial \boldsymbol{\Sigma}}\right|_{\boldsymbol{\Sigma}=\mathbf{\Sigma}^{*}} .
$$

Координаты неподвижных точек $\left(\Sigma_{1}, \Sigma_{2}\right)$, а также их собственные значения заданы в табл. 1-4. Динамический характер данной системы уравнений с неподвижными точками определяется соответствующей матрицей линеаризации. Действительные части собственных значений показывают, являются ли космологические решения репеллером (неустойчивыми), аттрактором (устойчивыми) или седловыми точками [101]. Динамические свойства неподвижной точки определяются знаком действительной части собственных значений. Если все собственные значения положительны, точка является репеллером; если все собственные значения отрицательны, точка является аттрактором; в противном случае мы имеем седловую точку. 
ТАБлицА 1. Неподвижные точки и собственные значения при $c=4, Q=1.45$, $\Omega_{\sigma 0}=0.001$ и $\lambda=-10^{-5}$.

\begin{tabular}{lc}
\hline \hline Координаты & Собственные значения \\
\hline$(1.41,4)$ & $8.33,2.88,0.006,-0.012,-0.336,-3.3$ \\
$(1.41,-4)$ & $2.88,0.012,-0.01,-0.335,-0.772,-1.91$ \\
$(-1.41,3.99)$ & $8.24,2.88,0.006,-0.011,-0.25,-3.32$ \\
$(-1.41,-3.99)$ & $8.24,3.32,2.88,0.25,0.011,0.006$ \\
\hline
\end{tabular}

ТАБлицА 2. Неподвижные точки и собственные значения при $c=4, Q=0.678$, $\Omega_{\sigma 0}=0.001$ и $\lambda=-10^{-5}$.

\begin{tabular}{ll}
\hline \hline Координаты & \multicolumn{1}{c}{ Собственные значения } \\
\hline$(1.41,4)$ & $2.96,1.48,0.699,0.1,-0.009,-0.012$ \\
$(1.41,-4)$ & $2.96,0.699,0.012,-0.009,-0.1,-1.48$ \\
$(1.806,4.338)$ & $6.78,3.09,-0.011,-0.013,-3.78,-9.08$ \\
$(1.806,-4.338)$ & $9.08,6.78,0.013,-0.011,-3.09,-3.78$ \\
$(-1.41,3.99)$ & $5.35,2.97,0.007,-0.011,-0.016,-2.73$ \\
$(-1.41,-3.99)$ & $5.35,2.97,2.73,0.016,0.011,0.007$ \\
\hline
\end{tabular}

ТАБлицА 3. Неподвижные точки и собственные значения при $c=10, Q=1.45$, $\Omega_{\sigma 0}=0.001$ и $\lambda=-10^{-5}$.

\begin{tabular}{ll}
\hline \hline Координаты & \multicolumn{1}{c}{ Собственные значения } \\
\hline$(0.8,4.21)$ & $2.8,1.1,-0.007,-0.012,-0.193,-4.23$ \\
$(0.8,-4.21)$ & $0.012,-0.007,-0.193,-1.1,-2.8,-4.23$ \\
$(1.41,6.32)$ & $2.88,0.493,0.489,-0.018,-0.01,-1.92$ \\
$(1.41,-6.32)$ & $2.88,0.018,-0.01,-0.49,-0.48,-1.92$ \\
$(-1.41,6.32)$ & $8.31,2.88,0.006,-0.018,-0.49,-2.09$ \\
$(-1.41,-6.32)$ & $8.31,2.88,2.09,0.493,0.018,0.006$ \\
\hline
\end{tabular}

ТАБлицА 4. Неподвижные точки и собственные значения при $c=10, Q=0.678$, $\Omega_{\sigma 0}=0.001$ и $\lambda=-10^{-5}$.

\begin{tabular}{ll}
\hline \hline Координаты & \multicolumn{1}{c}{ Собственные значения } \\
\hline$(1.41,6.32)$ & $2.96,0.939,0.686,0.12,-0.009,-0.018$ \\
$(1.41,-6.32)$ & $2.96,0.686,0.018,-0.009,-0.12,-0.939$ \\
$(1.8,6.8)$ & $6.76,1.98,-0.011,-0.02,-3.95,-14.73$ \\
$(1.8,-6.8)$ & $14.73,6.76,0.02,-0.011,-1.98,-3.95$ \\
$(-1.41,6.32)$ & $5.4,2.96,0.007,-0.018,-0.12,-1.71$ \\
$(-1.41,-6.32)$ & $5.4,2.96,1.71,0.12,0.018,0.007$ \\
\hline
\end{tabular}


ТАБлицА 5. Аттракторы, репеллеры и седловые точки; $\Omega_{\sigma 0}=0.001, \lambda=-10^{-5}$.

\begin{tabular}{lllccc}
\hline \hline Координаты & $c$ & $Q$ & Репеллер & Аттрактор & Седловая точка \\
\hline$(1.41,4)$ & 4 & 1.45 & - & - & Седло \\
$(1.41,-4)$ & 4 & 1.45 & - & - & Седло \\
$(-1.41,3.99)$ & 4 & 1.45 & - & - & Седло \\
$(-1.41,-3.99)$ & 4 & 1.45 & - & Аттрактор & - \\
$(1.41,4)$ & 4 & 0.678 & - & - & Седло \\
$(1.41,-4)$ & 4 & 0.678 & - & - & Седло \\
$(1.806,4.338)$ & 4 & 0.678 & - & - & Седло \\
$(1.806,-4.338)$ & 4 & 0.678 & - & - & Седло \\
$(-1.41,3.99)$ & 4 & 0.678 & - & - & Седло \\
$(-1.41,-3.99)$ & 4 & 0.678 & - & Аттрактор & - \\
$(0.8,4.21)$ & 10 & 1.45 & - & - & Седло \\
$(0.8,-4.21)$ & 10 & 1.45 & - & - & Седло \\
$(1.41,6.32)$ & 10 & 1.45 & - & - & Седло \\
$(1.41,-6.32)$ & 10 & 1.45 & - & - & Седло \\
$(-1.41,6.32)$ & 10 & 1.45 & - & - & Седло \\
$(-1.41,-6.32)$ & 10 & 1.45 & - & Аттрактор & - \\
$(1.41,6.32)$ & 10 & 0.678 & - & - & Седло \\
$(1.41,-6.32)$ & 10 & 0.678 & - & - & Седло \\
$(1.8,6.8)$ & 10 & 0.678 & - & - & Седло \\
$(1.8,-6.8)$ & 10 & 0.678 & - & - & Седло \\
$(-1.41,6.32)$ & 10 & 0.678 & - & Аттрактор & Седло \\
$(-1.41,-6.32)$ & 10 & 0.678 & - & & - \\
\hline
\end{tabular}

Теперь, используя уравнения (64)-(66), положим $c=4$ или $c=10$ и $Q=0.678$, $Q=1.45$ [15], $\Omega_{\sigma 0}=0.001, \lambda=-10^{-5}$, получим неподвижные точки (см. табл. $\left.1-4\right)$. Также мы определяем, являются ли точки, приведенные в табл. 1-4, репеллерами, аттракторами или седловыми точками (см. табл. 5). Важно отметить, что при $c>0$, $\lambda=-10^{-5}, \Omega_{\sigma 0}=0.001, Q=0.678$ и $Q=1.45$ [15] мы получаем как минимум аттрактор.

\section{9. ВЫВОДЫ И ОБСУЖДЕНИЯ}

В настоящей работе в рамках космологической модели ВI мы изучили модель дилатонного скалярного поля в пристуствии взаимодействия ДТЭ и ОДТЭ и ТМ, а также без него. Мы построили динамику модели дилатонного скалярного поля ТЭ согласно эволюционному поведению модели ТЭ со взаимодействием и без него. Кроме того, мы обсудили основные свойства параметра $\Omega_{\sigma 0}$ анизотропной энергии при различных значениях параметра взаимодействия $b^{2}$, которые можно суммировать следующим образом.

Во-первых, параметр уравнения состояния $\omega_{\Lambda}$ моделей ДТЭ и ОДТЭ в отсутствии взаимодействия не может пересечь фантомную разделительную линию и ведет себя 
как космологическая постоянная на поздних стадиях, в то время как параметр уравнения состояния $\omega_{\Lambda}$ в текущих ТЭ и ТМ в моделях ВI со взаимодействием может пересекать фантомную разделительную линию $\left(\omega_{\Lambda}<-1\right)$, как показано на рис. 1a, 5a. Такое поведение согласуется с текущими наблюдениями. Эволюция параметра плотности $\Omega_{\Lambda}$ ДТЭ и ОДТЭ с взаимодействием и без него зависит от параметра плотности анизотропии $\Omega_{\sigma 0}$. Во-вторых, для заданных постоянной взаимодействия $b^{2}$ и $\Omega_{\sigma 0}$ эволюция параметров плотности $\Omega_{\Lambda}$ ДТЭ и ОДТЭ возрастает вместе с возрастанием показателя масштаба. Также величина $\Omega_{\Lambda}$ возрастает при возрастании $b^{2}$ и убывает при возрастании $\Omega_{\sigma 0}$, как показано на рис. $16,5 б$. В-третьих, мы получили версии со взаимодействием и без взаимодействия ДТЭ и ОДТЭ для дилатонного скалярного поля в моделях ВI. Было изучено поведение кинетических энергий и динамика дилатонных скалярных полей при использовании надежных значений постоянных параметров. Результаты показаны на рис. 2, 6. Фантомное и обобщенное фантомное дилатонное скалярное поле для заданного $b^{2}$ или $\Omega_{\sigma 0}$ уменьшается при увеличении масштабирующего множителя, но кинетическая энергия возрастает. При заданном масштабирующем можителе фантомное дилатонное скалярное поле уменьшается и увеличивается при увеличении $b^{2}$ и $\Omega_{\sigma 0}$ соответственно, а кинетическая энергия растет и убывает с увеличением $b^{2}$ и $\Omega_{\sigma 0}$ соответственно. Анализ показывает, что эффекты, соответствующие взаимодействию ДТЭ и ОДТЭ, наблюдались на более поздних стадиях эволюции, чем в случае отсутствия и присутствия взаимодействия между компонентами.

Далее мы обсудили устойчивость модели относительно возмущений (см. рис. 3, 7). Было обнаружено, что на ранних стадиях модель остается неустойчивой, как и модели ТЭ [41], [102], в то время как на поздних стадиях возможно появление устойчивости. Кроме того, показано, что при увеличении параметра анизотропии устойчивость теории на поздних стадиях эволюции увеличивается. Также проверено, справедлив ли в данном сценарии обобщенный второй закон термодинамики (см. pис. 4,8$)$. Обнаружено, что $\dot{S}_{\text {tot }}>0$ в течение эволюции Вселенной. Это подтверждает корректность обобщенного второго закона термодинамики при построении взаимодействующих дилатонных ДТЭ и ОДТЭ в моделях ВI.

Наконец, мы исследовали динамическое поведение при расширении, которое ожидается в анизотропной Вселенной, по сравнению с изотропной Вселенной. На рис. 9 показана эволюция параметра Хаббла относительно красного смещения. Согласно данной модели ускоренное расширение Вселенной идет быстрее, чем в изотропной модели. Это означает, что чем больше анизотропия, тем более точное значение скорости расширения Хаббла $H(z)$ мы получаем. В этом случае параметр Хаббла ОДТЭ (ДТЭ) больше, чем во Вселенной $\Lambda$ CDM. Кроме того, мы изучили эволюцию $\sigma / H$ (см. рис. 10). Несмотря на то что сдвиг постоянен в течение периода анизотропной инфляции, он может уменьшится при изотропной инфляции. Мы показали, что анизотропия Вселенной $\sigma / H$ может возрастать вплоть до величин порядка $\mathcal{O}(1)$ в конце изотропной инфляции. Также был изучен рост структур в линейном режиме с эффектами анизотропии и показано, что рост возмущений плотности $g(a)$ замедляется в моделях $\Lambda$ CDM по сравнению с моделями ОДТЭ и ДТЭ (см. рис. 11).

Таким образом, заключаем, что в анизотропной Вселенной фактор роста меняется медленнее при увеличении параметра анизотропии, и он всегда меньше, чем во Вселенной ФРУ. Определена система дифференциальных уравнений первого по- 
рядка, которая описывает эволюцию двух безразмерных величин. Мы также проверили, что система принадлежит области квантовой устойчивости, показанной на рис. 12, в течение всей эволюции, это нам дает модель ТЭ, несмотря на то что даже коэффициент при члене $\dot{\phi}^{2}$ отрицательный. Показано, что дилатонное скалярное поле в модели ВІ имеет набор аттракторов (устойчивых точек) и седловых точек (см. табл. 5).

\section{Список литературы}

[1] A. G. Reiss, A. V. Filippenko, P. Challis et al., "Observational evidence from supernovae for an accelerating universe and a cosmological constant", Astron. J., 116:3 (1998), 1009-1038.

[2] S. Perlmutter, G. Aldering1, G. Goldhaber et al., "Measurements of $\Omega$ and $\Lambda$ from 42 high-redshift supernovae", Astrophys. J., 517:2 (1999), 565-586.

[3] D. N. Spergel, L. Verde, H. V. Peiris et al., "First-year Wilkinson Microwave Anisotropy Probe (WMAP) observations: determination of cosmological parameters", Astrophys. J. Suppl., 148:1 (2003), 175-194.

[4] D. N. Spergel, R. Bean, O. Doré et al., "Three-year Wilkinson Microwave Anisotropy Probe (WMAP) observations: implications for cosmology", Astrophys. J. Suppl., 170:2 (2007), $377-408$.

[5] C. Wetterich, "Cosmology and the fate of dilatation symmetry", Nucl. Phys. B, 302:4 (1988), 668-696.

[6] B. Ratra, P. J.E. Peebles, "Cosmological consequences of a rolling homogeneous scalar field", Phys. Rev. D, 37:12 (1988), 3406-3427.

[7] C. Armendariz-Picon, V.F. Mukhanov, P. J. Steinhardt, "Dynamical solution to the problem of a small cosmological constant and late-time cosmic acceleration", Phys. Rev. Lett., 85:21 (2000), 4438-4441.

[8] C. Armendariz-Picon, V.F. Mukhanov, P. J. Steinhardt, "Essentials of k-essence", Phys. Rev. D, 63:10 (2001), 103510, 13 pp.

[9] T. Padmanabhan, "Accelerated expansion of the universe driven by tachyonic matter", Phys. Rev. D, 66:2 (2002), 021301, 4 pp.

[10] A. Sen, "Tachyon matter", JHEP, 07 (2002), 065, 11 pp.

[11] R. R. Caldwell, "A phantom menace? Cosmological consequences of a dark energy component with super-negative equation of state", Phys. Lett. B, 545:1-2 (2002), 23-29.

[12] S. Nojiri, S.D. Odintsov, "Quantum de Sitter cosmology and phantom matter", Phys. Lett. B, 562:3-4 (2003), 147-152.

[13] S. Nojiri, S. D. Odintsov, "de Sitter brane universe induced by phantom and quantum effects", Phys. Lett. B, 565 (2003), 1-9.

[14] N. Arkani-Hamed, H.-C. Cheng, M. A. Luty, S. Mukohyama, "Ghost condensation and a consistent infrared modification of gravity", JHEP, 05 (2004), 074, 37 pp.

[15] F. Piazza, S. Tsujikawa, "Dilatonic ghost condensate as dark energy", J. Cosmol. Astropart. Phys., 07 (2004), 004, 26 pp.

[16] E. Elizalde, S. Nojiri, S. D. Odintsov, "Late-time cosmology in a (phantom) scalar-tensor theory: dark energy and the cosmic speed-up", Phys. Rev. D, 70:4 (2004), 043539, 20 pp.

[17] S. Nojiri, S. D. Odintsov, S. Tsujikawa, "Properties of singularities in the (phantom) dark energy universe", Phys. Rev. D, 71:6 (2005), 063004, 16 pp.

[18] A. Anisimov, E. Babichev, A. Vikman, "B-inflation", J. Cosmol. Astropart. Phys., 06 (2005), 006, 23 pp.

[19] E. Witten, "The cosmological constant from the viewpoint of string theory", Sources and Detection of Dark Matter and Dark Energy in the Universe (Marina del Rey, CA, USA, February 23-25, 2000), ed. D. B. Cline, Springer, Berlin, Heidelberg, 2001, 27-36. 
[20] R.-G. Cai, "A dark energy model characterized by the age of the Universe", Phys. Lett. B, 657:4-5 (2007), 228-231.

[21] H. Wei, R.-G. Cai, "A new model of agegraphic dark energy", Phys. Lett. B, 660:3 (2008), $113-117$.

[22] E. J. Copeland, M. Sami, S. Tsujikawa, "Dynamics of dark energy", Internat. J. Modern Phys. D, 15:11 (2006), 1753-1935.

[23] M. Li, X.-D. Li, S. Wang, Y. Wang, "Dark energy", Commun. Theor. Phys., 56:3 (2011), 525-604.

[24] A. De Felice, S. Tsujikawa, " $f(R)$ theories", Living Rev. Relativ., 13 (2010), 3-161.

[25] S. Capozziello, M. de Laurentis, "Extended theories of gravity", Phys. Rep., 509:4-5 (2011), $167-320$.

[26] T. Clifton, P. G. Ferreira, A. Padilla, C. Skordis, "Modified gravity and cosmology", Phys. Rep., 513:1-3 (2012), 1-189.

[27] S. Nojiri, S. D. Odintsov, "Unified cosmic history in modified gravity: from $F(R)$ theory to Lorentz non-invariant models", Phys. Rep., 505:2-4 (2011), 59-114.

[28] G. Dvali, G. Gabadadze, M. Porrati, "4D gravity on a brane in 5D Minkowski space", Phys. Lett. B, 485:1-3 (2000), 208-214.

[29] M.S. Carena, J. Lykken, M. Park, J. Santiago, "Self-acclerating warped braneworlds", Phys. Rev. D, 75:2 (2007), 026009, 17 pp.

[30] M. Minamitsuji, "Self-accelerating solutions in the cascading DGP braneworld", Phys. Lett. B, 684:2-3 (2010), 92-95.

[31] A. Sheykhi, B. Wang, N. Riazi, "String inspired explanation for the superacceleration of our Universe", Phys. Rev. D, 75:12 (2007), 123513, 8 pp.

[32] F. R. Urban, A. R. Zhitnitsky, "Cosmological constant from the ghost: a toy model", Phys. Rev. D, 80:6 (2009), 063001, 13 pp.

[33] N. Ohta, "Dark energy and QCD ghost", Phys. Lett. B, 695:1-4 (2011), 41-44.

[34] F. R. Urban, A. R. Zhitnitsky, "Cosmological constant, violation of cosmological isotropy and CMB", J. Cosmol. Astropart. Phys., 09 (2009), 018, 14 pp.

[35] E. Witten, "Current algebra theorems for the $U(1)$ 'Goldstone boson'", Nucl. Phys. B, 156:2 (1979), 269-283.

[36] F. R. Urban, A. R. Zhitnitsky, "The cosmological constant from the QCD Veneziano ghost", Phys. Lett. B, 688:1 (2010), 9-12.

[37] G. Veneziano, "U(1) without instantons", Nucl. Phys. B, 159:1-2 (1979), 123-224.

[38] F. R. Urban, A. R. Zhitnitsky, "The QCD nature of dark energy", Nucl. Phys. B, 835:1-2 (2010), 135-173.

[39] A.R. Zhitnitsky, "Contact term, its holographic description in QCD and dark energy", Phys. Rev. D, 86:4 (2012), 045026, 14 pp.

[40] M. Maggiore, "Zero-point quantum fluctuations and dark energy", Phys. Rev. D, 83:6 (2011), 063514, 16 pp.

[41] E. Ebrahimi, A. Sheykhi, Internat. J. Modern Phys. D, 20:12 (2011), 2369-2381.

[42] A. Sheykhi, "Holographic scalar field models of dark energy", Phys. Rev. D, 84:10 (2011), 107302, 5 pp.

[43] A. Sheykhi, "Thermodynamics of interacting holographic dark energy with the apparent horizon as an IR cutoff", Class. Quantum Grav., 27:2 (2010), 025007, 8 pp.; M. Mazumder, S. Chakraborty, "Validity of the generalized second law of thermodynamics of the universe bounded by the event horizon in holographic dark energy model", Gen. Rel. Grav., 42:4 (2010), 813-820.

[44] M. Sharif, M. Zubair, "Thermodynamics in $f(R, T)$ theory of gravity", J. Cosmol. Astropart. Phys., 03 (2012), 028; Erratum, 05 (2012), E01, arXiv: 1204.0848; "Thermodynamic behavior of particular $f(R, T)$-gravity models", KЭЭTФ, 144:2(8), 291-300. 
[45] K. Karami, A. Abdolmaleki, "Generalized second law of thermodynamics in $f(T)$ gravity", J. Cosmol. Astropart. Phys., 04 (2012), 007, arXiv: 1201.2511.

[46] H. K. Eriksen, F. K. Hansen, A. J. Banday, K. M. Górski, P. B. Lilje, "Asymmetries in the cosmic microwave background anisotropy field", Astrophys. J., 605:1 (2004), 14-20.

[47] T. R. Jaffe, A. J. Banday, H. K. Eriksen, K. M. Górski, F. K. Hansen, "Fast and efficient template fitting of deterministic anisotropic cosmological models applied to WMAP data", Astrophys. J., 643:2 (2006), 616-629.

[48] L. Campanelli, P. Cea, L. Tedesco, "Ellipsoidal universe can solve the cosmic microwave background quadrupole problem", Phys. Rev. Lett., 97:13 (2006), 131302, 4 pp.; Erratum, Phys. Rev. Lett., 97:20 (2006), 209903.

[49] B. Saha, "Anisotropic cosmological models with perfect fluid and dark energy", Chinese J. Phys., 43:6 (2005), 1035-1043, arXiv: gr-qc/0412078.

[50] K.S. Adhav, "Binary mixture of anisotropic dark energy and perfect fluid in Kantowski-Sach universe", Eur. Phys. J. Plus, 126 (2011), 103, 8 pp.

[51] G. P. Singh, Binaya K. Bishi, P. K. Sahoo, "Bianchi type-I bulk viscous cosmology with Chaplygin gas in Lyra geometry", Chinese J. Phys., 54:6 (2016), 895-905.

[52] Shri Ram, S. Chandel, M. K. Verma, "Kantowski-Sachs universe with anisotropic dark energy in Lyra geometry", Chinese J. Phys., 54:6 (2016), 953-959.

[53] H. Hossienkhani, "Bianchi type I universe and interacting ghost scalar fields models of dark energy", Astrophys. Space Sci., 361:4 (2016), 136, 6 pp.

[54] G. P. Singh, Binaya K. Bishi, P.K. Sahoo, "Scalar field and time varying cosmological constant in $f(R, T)$ gravity for Bianchi type-I universe", Chinese J. Phys., 54:2 (2016), $244-255$.

[55] S. D. Katore, A. Y. Shaikh, A. S. Bhaskar, "Anisotropic dark energy cosmological models from early deceleration to late time acceleration in Lyra geometry", Bulg. J. Phys., 41:1 (2014), 34-59.

[56] S. D. Katore, S. P. Hatkar, "Kaluza Klein universe with magnetized anisotropic dark energy in general relativity and Lyra manifold", New Astronomy, 34 (2015), 172-177.

[57] A. Pradhan, A. K. Vishwakarma, "A new class of LRS Bianchi type-I cosmological models in Lyra geometry", J. Geom. Phys., 49:3-4 (2004), 332-342.

[58] F. Rahaman, N. Begum, G. Bag, B. C. Bhui, "Cosmological models with negative constant deceleration parameter in Lyra geometry", Astrophys. Space Sci., 299:3 (2005), 211-218.

[59] N. Azimi, F. Barati, "Instability of interacting ghost dark energy model in an anisotropic universe", Internat. J. Theor. Phys., 55:7 (2016), 3318-3328.

[60] F. Barati, "Interacting generalized ghost dark energy in non-isotropic background", Internat. J. Theor. Phys., 55:4 (2016), 2189-2198.

[61] M. Gasperini, F. Piazza, G. Veneziano, "Quintessence as a runaway dilaton", Phys. Rev. D, 65:2 (2002), 023508, 13 pp.

[62] M. Gasperini, R. Ricci, "Homogeneous conformal string backgrounds", Class. Quantum Grav., 12:3 (1995), 677-699; M. Gasperini, "Dilatonic interpretation of quintessence?", Phys. Rev. D, 64:4 (2001), 043510, 7 pp.

[63] R. H. Brandenberger, R. Easther, J. Maia, "Nonsingular dilaton cosmology", JHEP, 08 (1998), 007, 15 pp.

[64] C. Armendáriz-Picón, T. Damour, V. Mukhanov, "k-Inflation", Phys. Lett. B, 458:2-3 (1999), 209-218.

[65] N. Arkani-Hamed, P. Creminelli, S. Mukohyama, M. Zaldarriaga, "Ghost inflation", J. Cosmol. Astropart. Phys., 04 (2004), 001, 19 pp.

[66] O. Bartolami, F. Gil Pedro, M. Le Delliou, "Dark energy-dark matter interaction and putative violation of the equivalence principle from the Abell cluster A586", Phys. Lett. B, 654:5-6 (2007), 165. 
[67] B. Wang, Y. Gong, E. Abdalla, "Transition of the dark energy equation of state in an interacting holographic dark energy model", Phys. Lett. B, 624:3004 (2005), 141-146.

[68] A. A. Sen, D. Pavón, "Reconstructing the interaction rate in holographic models of dark energy", Phys. Lett. B, 664:1-2 (2008), 7-11.

[69] P. J.E. Peebles, B. Ratra, "The cosmological constant and dark energy", Rev. Modern Phys., 75:2 (2003), 559-606.

[70] J. D. Bekenstein, "Black holes and entropy", Phys. Rev. D, 7:8 (1973), 2333-2346.

[71] E. N. Saridakis, P. F. González-Díaz, C. L. Sigüenza, "Unified dark energy thermodynamics: varying $w$ and the -1-crossing", Class. Quantum Grav., 26:16 (2009), 165003, 8 pp.

[72] M. Jamil, E. N. Saridakis, M. R. Setare, "Thermodynamics of dark energy interacting with dark matter and radiation", Phys. Rev. D, 81:2 (2010), 023007, 6 pp.

[73] H. Wei, "Entropy-corrected holographic dark energy", Commun. Theor. Phys., 52:4 (2009), 743-749.

[74] S.H. Pereira, J.F. Jesus, "Can dark matter decay in dark energy?", Phys. Rev. D, 79:4 (2009), 043517, 6 pp.

[75] R.-G. Cai, Z.-L. Tuo, Y.-B. Wu, Y.-Y. Zhao, "More on QCD ghost dark energy", Phys. Rev. D, 86:2 (2012), 023511, 8 pp.

[76] A. Sheykhi, M. Sadegh Movahed, "Interacting ghost dark energy in non-flat universe", Gen. Rel. Grav., 44:2 (2012), 449-465.

[77] R. Jimenez, A. Loeb, "Constraining cosmological parameters based on relative galaxy ages", Astrophys. J., 573:1 (2002), 37-42.

[78] T. M. Davis, E. Mörtsell, J. Sollerman et al., "Scrutinizing exotic cosmological models using ESSENCE supernova data combined with other cosmological probes", Astrophys. J., 666:2 (2007), 716-725.

[79] E. Komatsu, J. Dunkley, M. R. Nolta et al., "FIVE-year Wilkinson Microwave Anisotropy Probe observations: cosmological interpretation", Astrophys. J. Suppl., 180:2 (2009), 330-376.

[80] P. A. R. Ade, N. Aghanim, C. Armitage-Caplan et al. [Planck Collab.], "Planck 2013 results. XVI. Cosmological parameters", Astron. Astrophys., 571 (2014), A16, 66 pp., arXiv: 1303.5076.

[81] W. J. Percival, S. Cole, D. J. Eisenstein, R. C. Nichol, J. A. Peacock, A. C. Pope, A. S. Szalay, "Measuring the baryon acoustic oscillation scale using the Sloan Digital Sky Survey and 2dF Falaxy Redshift Survey", Monthly Notices Roy. Astron. Soc., 381:3 (2007), 1053-1066.

[82] A. G. Riess, L. Macri, S. Casertano, M. Sosey, H. Lampeitl, H. C. Ferguson, A. V. Filippenko, S. W. Jha, W. Li, R. Chornock, D. Sarkar, "A redetermination of the Hubble constant with the Hubble Space Telescope from a differential distance ladder", Astrophys. J., 699:1 (2009), 539-563.

[83] Y.-S. Song, W. J. Percival, "Reconstructing the history of structure formation using redshift distortions", J. Cosmol. Astropart. Phys., 10 (2009), 004, 20 pp.

[84] A. G. Riess, L. Macri, S. Casertano, H. Lampeitl, H. C. Ferguson, A. V. Filippenko, S. W. Jha, W. Li, R. Chornock, "A 3\% solution: determination of the Hubble constant with the Hubble Space Telescope and Wide Field Camera 3", Astrophys. J., 730:2 (2011), 119, 18 pp.

[85] G. Chen, J. R. Gott III, B. Ratra, "Non-Gaussian error distribution of Hubble constant measurements", Publ. Astron. Soc. Pac., 115:813 (2003), 1269-1279, arXiv: astro-ph/0308099.

[86] J. R. Gott III, M.S. Vogeley, S. Podariu, B. Ratra, "Median statistics, $H_{0}$, and the accelerating universe", Astrophys. J., 549:1 (2001), 1-17.

[87] M. Li, X.-D. Li, S. Wang, X. Zhang, "Holographic dark energy models: a comparison from the latest observational data", J. Cosmol. Astropart. Phys., 06 (2009), 036, 16 pp.

[88] J. Simon, L. Verde, R. Jimenez, "Constraints on the redshift dependence of the dark energy potential", Phys. Rev. D, 71:12 (2005), 123001, 18 pp. 
[89] C. Blake, S. Brough, M. Colless et al., "The WiggleZ Dark Energy Survey: joint measurements of the expansion and growth history at $z<1$ ", Monthly Notices Roy. Astron. Soc., 425:1 (2012), 405-414.

[90] Y.-L. Li, S.-Y. Li, T.-J. Zhang, T.-P. Li, "Model-independent determination of curvature parameter using $H(z)$ and $D_{A}(z)$ data pairs from BAO measurements", Astrophys. J. Lett., 789:1 (2014), L15, 5 pp.

[91] P. K. Aluri, S. Panda, M. Sharma, S. Thakur, "Anisotropic universe with anisotropic sources", J. Cosmol. Astropart. Phys., 12 (2013), 003.

[92] F. Pace, J. C. Waizmann, M. Bartelmann, "Spherical collapse model in dark-energy cosmologies", Monthly Notices Roy. Astron. Soc., 406:3 (2010), 1865-1874.

[93] F. Pace, L. Moscardini, R. Crittenden, M. Bartelmann, V. Pettorino, "A comparison of structure formation in minimally and non-minimally coupled quintessence models", Monthly Notices Roy. Astron. Soc., 437:1 (2014), 547-561.

[94] W. J. Percival, "Cosmological structure formation in a homogeneous dark energy background", Astron. Astrophys., 443:3 (2005), 819-830.

[95] O. Bertolami, R. Lehnert, R. Pottin, A. Ribeiro, "Cosmological acceleration, varying couplings, and Lorentz breaking", Phys. Rev. D, 69:8 (2004), 083513, 11 pp.

[96] И. Я. Арефьева, Н. В. Булатов, С. Ю. Вернов, "Стабильные точные решения в космологических моделях с двумя скалярными полями", ТМФ, 163:3 (2010), 475-494.

[97] I. Ya. Aref'eva, N. V. Bulatov, L. V. Joukovskaya, S. Yu. Vernov, "Null energy condition violation and classical stability in the Bianchi I metric", Phys. Rev. D, 80:8 (2009), 083532, $13 \mathrm{pp}$.

[98] I. Ya. Aref'eva, A. S. Koshelev, S. Yu. Vernov, "Crossing the $w=-1$ barrier in the D3-brane dark energy model", Phys. Rev. D, 72:6 (2005), 064017, 11 pp.

[99] P. G. Ferreira, M. Joyce, "Cosmology with a primordial scaling field", Phys. Rev. D, 58:2 (1998), 023503, 23 pp.; E. J. Copeland, A. R. Liddle, D. Wands, "Exponential potentials and cosmological scaling solutions", Phys. Rev. D, 57:8 (1998), 4686-4690.

[100] M. R. Setare, E. C. Vagenas, "The cosmological dynamics of interacting holographic dark energy model", Internat. J. Modern Phys. D, 18:1 (2009), 147-157.

[101] D. Iakubovskyi, Y. Shtanov, "Braneworld cosmological solutions and their stability", Class. Quantum Grav., 22:12 (2005), 2415-2432.

[102] Y.S. Myung, "Instability of holographic dark energy models", Phys. Lett. B, 652:5-6 (2007), 223-227; K. Y. Kim, H. W. Lee, Y. S. Myung, "Instability of agegraphic dark energy models", Phys. Lett. B, 660:3 (2008), 118-124.

Поступила в редакцию 16.12.2016, после доработки 29.03.2017 